

Calhoun: The NPS Institutional Archive DSpace Repository

\title{
An examination of microthermal structure statistics as calculated from expendable bathythermograph records.
}

Hunt, Frank Martin Jr.

Monterey, California. Naval Postgraduate School

http://hdl.handle.net/10945/15704

This publication is a work of the U.S. Government as defined in Title 17, United States Code, Section 101. Copyright protection is not available for this work in the United States.

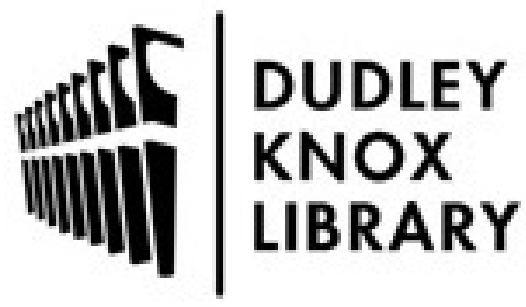

http://www.nps.edu/library
Calhoun is the Naval Postgraduate School's public access digital repository for research materials and institutional publications created by the NPS community. Calhoun is named for Professor of Mathematics Guy K. Calhoun, NPS's first appointed -- and published -- scholarly author.

Dudley Knox Library / Naval Postgraduate School 411 Dyer Road / 1 University Circle Monterey, California USA 93943 


\section{AN EXAMINATION OF MICROTHERMAL STRUCTURE STATISTICS AS CALCULATED FROM EXPENDABLE BATHYTHERMOGRAPH RECORDS}

FRANK MARTIN HUNT 
<smiles></smiles> 




\section{United States \\ Naval Postgraduate School

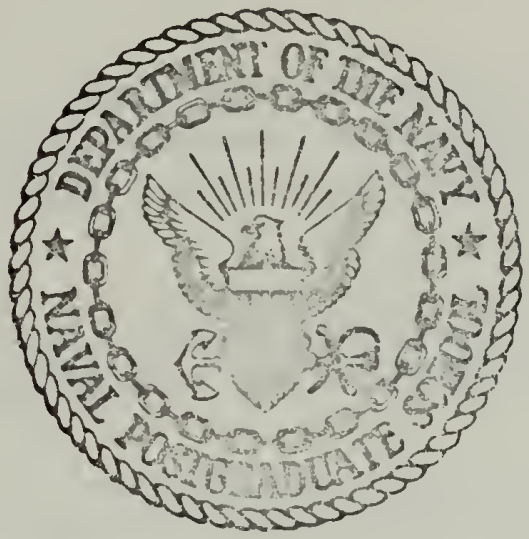

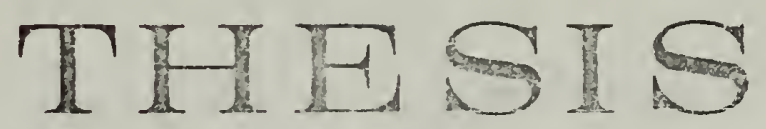

AN EXAMINATION OE

MICROTHERMAL STRUCTURE STATISTIICS

AS CALCULATED FROMI

EXPENDABLE BATHYTHERMOGPAPH RECORDS

by

Frank Martin Hunt, Jr.

Thesis Advisor

W.W. Denner

September 1971

Approved for public release; distribution unlimited. 
H 9428

c. 1 
An Examination of Microthermal Structure Statistics as

Calculated from Expendable Bathythermograph Records

$$
\text { by }
$$

Frank Martin Hunt, Jr.

Lieutenant Commander, United States Navy

B.S., United States Naval Academy, 1960

Submitted in partial fulfillment of the requirements for the degree of

MASTER OF SCIENCE IN OCEANOGRAPHY

from the

NAVAL POSTGRADUATE SCHOOL

September $197 i$ 



\section{ABSTRACT}

Expendable Bathythermograph (XBT) temperature depth profile data from 17 locations in the north Pacific Ocean has been analyzed to extract information concerning the thermal microstructure. The microstructure information is then examined to determine if the distribution can be associated with the climatic regions of the north Pacific Ocean proposed by Tully [ 1964 ].

A modified binomial filter used by Roden [ 1971 ] to analyze STD data was applied to find a mean temperature series. A temperature perturbation series results when the mean is subtracted from the original XBT series. Two resulting parameters, avexage mean temperature perturbation vertical thickness scale and average maximum amplitude of the temperature perturbations, can be associated with the microstructure.

The average mean temperature perturbation vertical thickness scale was found to increase with latitude from $20^{\circ} \mathrm{N}$ to $55^{\circ} \mathrm{IN}$, and as the thickness scale increased, the maximum amplitude of the perturbations was found to decrease. 
TABLE OF CONTENTS

I. INTRODUCTION -- 10

II. THEORY OF ACOUSTIC PROPAGATION IN A RANDOMLY

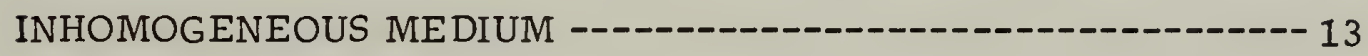

III. RECENT MEASUREMENTS OF MICROSTRUCTURE -------------24

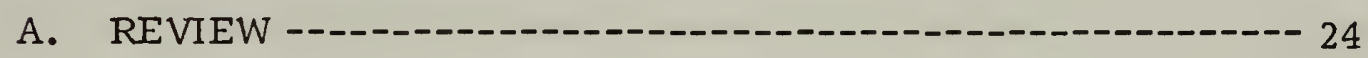



1. Insolation - 33

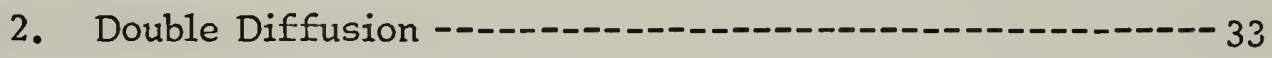

3. Internal Waves ---

4. Current Shear ---

IV. INFORMATION CONCERNING THE THERMAL IMICROSTRUCTURE THAT CAN BE GATHERED FROM EXISTING EXPENDABLE

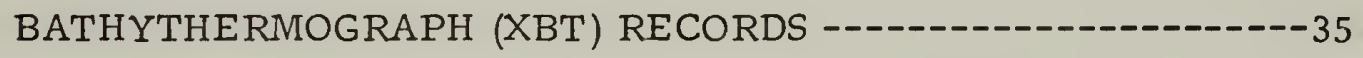

A. AVAILABLE DATA -

B. EXPENDABLE BATHYTHERIMOGRAPH SYSTEM ------------35

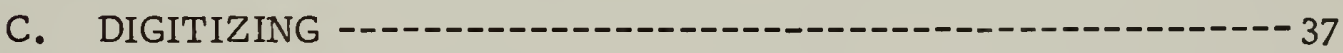

D. THERIMAL MICROSTRUCTURE ---------------------- 4 I

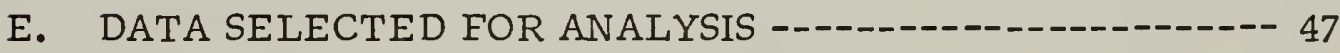

F. DESCRIPTION OF MICROSTRUCTURE ON XBT TRACE ----- 48 

G. OCEANOGRAPHIC REGIONS OF THE NORTH PACIEIC

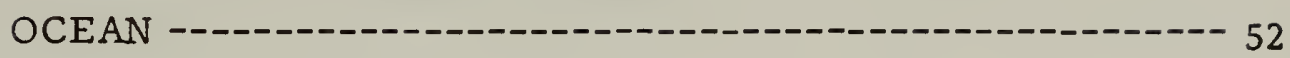

1. Description --- 52

2. Microstructure Distribution -- 55

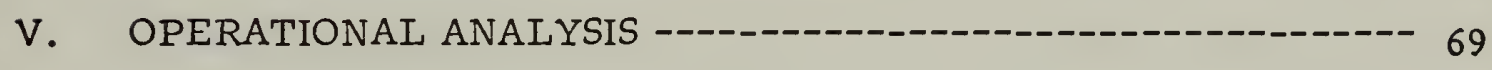

A. OPERATIONAL REQUIREMENT --1-- 69

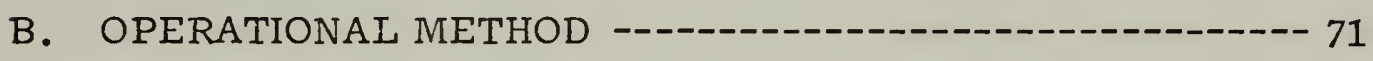

C. POSSIBLE UTILIZATION OF MICROSTRUCTURE

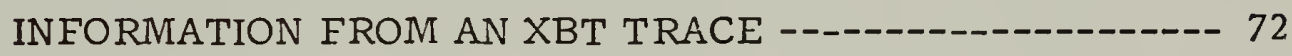

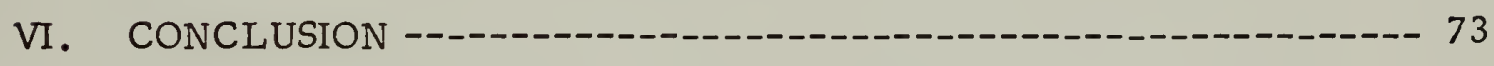

A. SUMMARY --

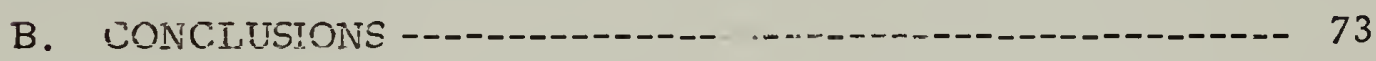

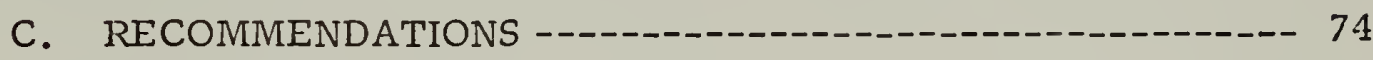

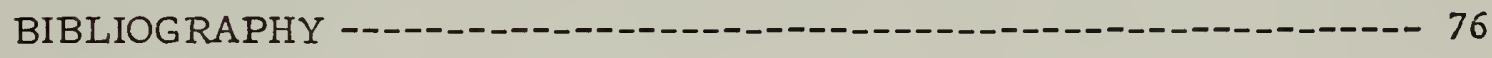

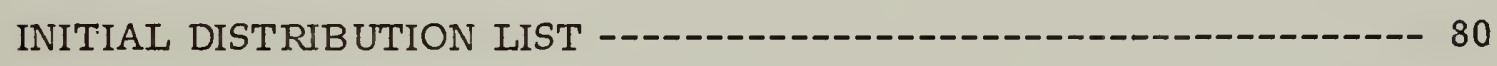

FORM DD 1473 -- 



\section{LIST OF TABLES}

I. Specifications of XBT System - 36

II. Subtropic Region -- 61

III. Subarctic Region -- 62

IV. Transport Regions -- 63

V. Other Regions -- 64

VI. California Current Time Series - 65 



\section{LIST OF FIGURES}

1. Coefficients of Variation of the amplitudes of direct signals plotted against the range to the receiver. Sound frequency,

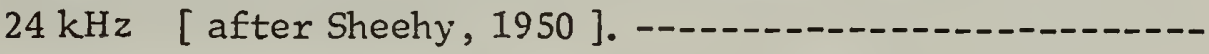

2. Focusing Range (meters) as a Function of Frequency ( $\mathrm{kHz}$ )

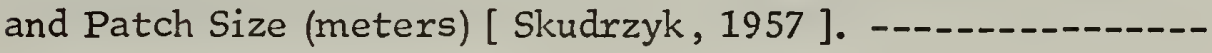

3. Increase in focusing due to spheriodal shape of a patch as a function of the ratio of the lengths of the vertical (b) to horizontal (a) axis [ Whitmarsh, Skudrzyk, and Urick, 1957 ].- 20

4. Illustration of the various types of acoustic scattering due to a thermal patch in the sea [Whitmarsh, Skudrzyk, and Urick, 1957 ].

5. Horizontal temperature distributions as measured in the sea [ after Whitmarsh, Skudrzyk, and Urick, 1957 ].---.---.----

6. Temperature and temperature-gradient sowndings for 0630 24 August 1966 in the Mediterranean Sea [Woods, 1968a ]. --

7. Temperature and temperature-gradient soundings for Maltese waters, 14 September 1967 [ Woods, 1968b ]. --.-.---

8. Corrected data from the upper portion of the water column in the San Diego Trough on 18 September 1968. The curve on the right is the continuation of the curve on the left [Osborn, 1969 ].

9. Vertical profile of temperature under $\mathrm{T}-3\left(84^{\circ} 38^{\prime} \mathrm{N}\right.$, $128^{\circ} 21.6 \% \mathrm{~W}, 19$ March 1969). Shaded section of profile (a) shown as observed in profile (b) to left [ Neshyba, Neal, and Denner, 1969 ].

10. Time series taken from $\mathrm{T}-3$ between $2145 \mathrm{Z} 17$ March and 2033218 March 1969. Temperature increases from right to left [ Neshyba, Neal, and Denner, 1969 ].

11. Unretouched Bathysonde profiles from the western Irish Sea of temperature and conductivity showing pronounced microstructure [ Simpson, 1971 ].-...-...-....-.-.-- 
12. Digitized record [Dale and Stevens, 1970].

13. Comparison of the original XBT trace with the trace reconstructed from the digitized data. The digitized trace is plotted to a scale of 90 meters/in due to computer requirements while the original trace is scaled to 92.3 meters/in. --

14. Computer solution for modified binomial filter response with filtering intervals of 1,2 , and 3 meters.

15. Result of modified binomial filter applied to Theoretical XBT trace. From left to right, Theoretical XBT trace, Mean curve, and Temperature Perturbation curve for a 7 meter wave length, $0.5^{\circ} \mathrm{C}$ Temperature variation, and a 1 meter filtering interval.

16. Result of modified binomial filter applied to theoretical XBT trace showing the amplification in the temperature perturbation amplitude when the wave length of the perturbation is on the slope part of the response curve of Figure 14. From left to right, Theoretical XBT trace, Mcan curve, and Temperature Perturbation curve for a 34 meter wave length, $0.5^{\circ} \mathrm{C}$ Temperature variation, and a 1 meter filtering interval.

17. Composite diagram of the original XBT trace, the reconstructed Digitized trace, the Mean curve found by applying Roden's filter, and the Temperature Perturbation curve. The original trace is scaled to 92.3 meters/in while the remaining curves are scaled 90.0 meters/in. --.--

18. Temperature perturbation curves for a filtering interval of 1,2 , and 3 meters. -------

19. Regions of the North Pacific Ocean [Tully, 1964].

20. Drift in the North Pacific Ocean [Tully, 1964]. -----.----

21. Plot of the Average Mean Temperature Perturbation Vertical Thickness Scale versus Latitude showing an increase in the vertical thickness scale for an increase in latitude between $20^{\circ} \mathrm{N}$ and $55^{\circ} \mathrm{N}$. ( $\Delta$, points along Longitude $156-157^{\circ} \mathrm{W}$; : , all other points). ------------ 
22. Plot of the Average Maximum Amplitude of the Temperature Perturbations versus Latitude showing the Amplitude decreases with an increase in latitude between $20^{\circ} \mathrm{N}$ and $55^{\circ} \mathrm{N}$. ( $\Delta$, points along Longitude $156-157^{\circ} \mathrm{W} ; \cdot$, all other points). --

23. Plot showing the Average Maximum Amplitude of the Temperature Perturbations decreases as the Average Mean Temperature Perturbation Vertical Thickness Scale increases between $20^{\circ} \mathrm{N}$ and $55^{\circ} \mathrm{N}$. ( $\Delta$, points along Longitude $156-157^{\circ} \mathrm{W}$; , all other points). 



\section{ACKNOWLEDGEMENTS}

The author wishes to acknowledge with gratitude the guidance, patience, and understanding provided by Associate Professor W. W. Denner during the preparation of this thesis.

The generous donation by Dr. N.E.J. Boston of his time and advice was essential for the orderly completion of this thesis.

Special thanks are extended to Mr. Dean Dale and Mr. Paul Stevens of Fleet Numerical Weather Central, Monterey, California for their kind cooperation in providing data and assistance whenever requested.

A final but most sincere word of appreciation is extended to Mr.

j. F.T. Saur of the Fishery-Oceanography Group of the United States

Department of Commerce, National Oceanic and Atmospheric Administration, National Marine Fisheries Service in La Jolla, California for providing copies of original XBT traces. 



\section{INTRODUCTION}

Temperature and salinity distribucions in the ocean determine the propagation paths for acoustical energy. The gross temperature structure is used for ray path calculations to predict transmission characteristics [Cohen and Weinberg, 1969]. However, sound measurements at one location reveal a fluctuating accustic pressure level [Sheehy, 1950] attributed to variations in the fine temperature and salinity structure that cause small but important changes in the propagation paths. At higher frequencies the microstructure becomes increasingly significant. A theory for acoustic wave transmission has been developed for a medium containing thermal microstructure assuming that the microstructure can be represented by inhomogeneities of a thermal nature that are spherical or spheroidal "patches" randomly distributed in space [For example, Liebermann, 1951; Mintzer, 1953a, 1953b, 1954]. The measured sound fluctuations have been related to the focusing or defocusing of acoustic waves by these "patches" at ranges less than $\mathrm{ka}^{2}$ (where $\mathrm{k}$ is the acoustic nave number and $\mathrm{a}$ is the effective radius of the patch) and to interference at ranges greater than $\mathrm{ka}^{2}$ [Skudrzyk, 1957].

Recent findings conceming the microstructure do not support this "patch" distribution assumption. A layered microstructure with layers of small gradient a few to a few tens of meters thick separated by sheets of. high gradient a few to a few tens of centimeters thick nd with 

horizontal dimensions up to thousands of meters have been found in many regions of the oceans [Cooper, 1967; Cooper and Stomme1, 1968; Neshyba, Neal, and Denner, 1969; Osborn, 1969; Roden, 1971; Simpson, 1971;

Stomme1 and Fedorov, 1967; and Woods, 1968]. Several mechanisms have been proposed for generating the layered microstructure, and it may be that a different mechanism is dominant for different ocean regions. This layered microstructure concept requires a re-examination of the present theory for the transmission of acoustic energy in a randomly inhomogeneous medium.

In 1957, Whitmarsh, Skudrzyk, and Urick concluded a paper on the scattering of sound in the sea with the following comment: "... the greatest gap seems to lie in the microthermal statistics of the ocean. If these were known better than they are now, apparently the amplitude statistics of the fluctuations of sound transmitted through the sea could be predicted roughly, but with some assurance. "I Some 11 years later, during a meeting on Turbulence In The Ocean held at The University of British Columbia, R. W. Stewart "pointed out that a case existed for another degree of exploration of the ocean. While in the past, widespread surveys have sought to measure the temperature and salinity fields, we must now concentrate on a widespread search for fluctuations in the

1 Whitmarsh, D.C., Skudrzyk, E., and Urick, R.J ., "Forward Scattering of Sound in the Sea and I ts Correl ati on with Temperature Microstructure," I. Acous. Soc. Am., v. 29, p. 1141, October 1957. 

temperature and salinity fields. ${ }^{2}$ The only data that exists with such broad coverage is bathythermograph data. Magruder [1970] suggests that Expendable Bathythermograph (XBT) data might be useful.

The purpose of this thesis is to see if information about the distribution of microstructure can be determined from XBT traces. If the microstructure distribution can be determined from an XBT trace, then using some of this available data, can the distribution be related to the climatic regions of the ocean for use as a rational basis for assessing the acoustic variability in these regions? Finally, what operational techniques can be developed using knowledge of the microstructure determined from the XBT trace that would be of benefit to the Navy in the operating environment?

Tens of thousands of XBT traces are on file at the National Oceanographic Data Center (NODC), with the number increasing each day. Under the Fleet Numerical Weather Central (FNWC), Cooperative Oceanographic Observation Program, XBT traces are also available from commercial carrier trans-atlantic and trans-pacific routes. While the distribution is by no means uniform in space or time, it is the only source of microstructure data covering the world oceans.

2 Osborn, T.R., Report of the Symposium on Turbulence in the Ocean, Institute of Oceanography, University of British Columbia, Vancouver, British Columbia, p. 20, July 1970. 


\section{THEORY OF ACOUSTIC PROPAGATION IN A RANDOMLY INHOMOGENEOUS MEDIUM}

Sheehy [1950] published data showing that the magnitude of the acoustic pressure maximum amplitude fluctuations (Coefficient of Variation) for $24 \mathrm{kHz}$ direct path transmissions increased proportional to the square root of the range. However, it should be noted from Eigure 1 that the scatter of data is rather large.

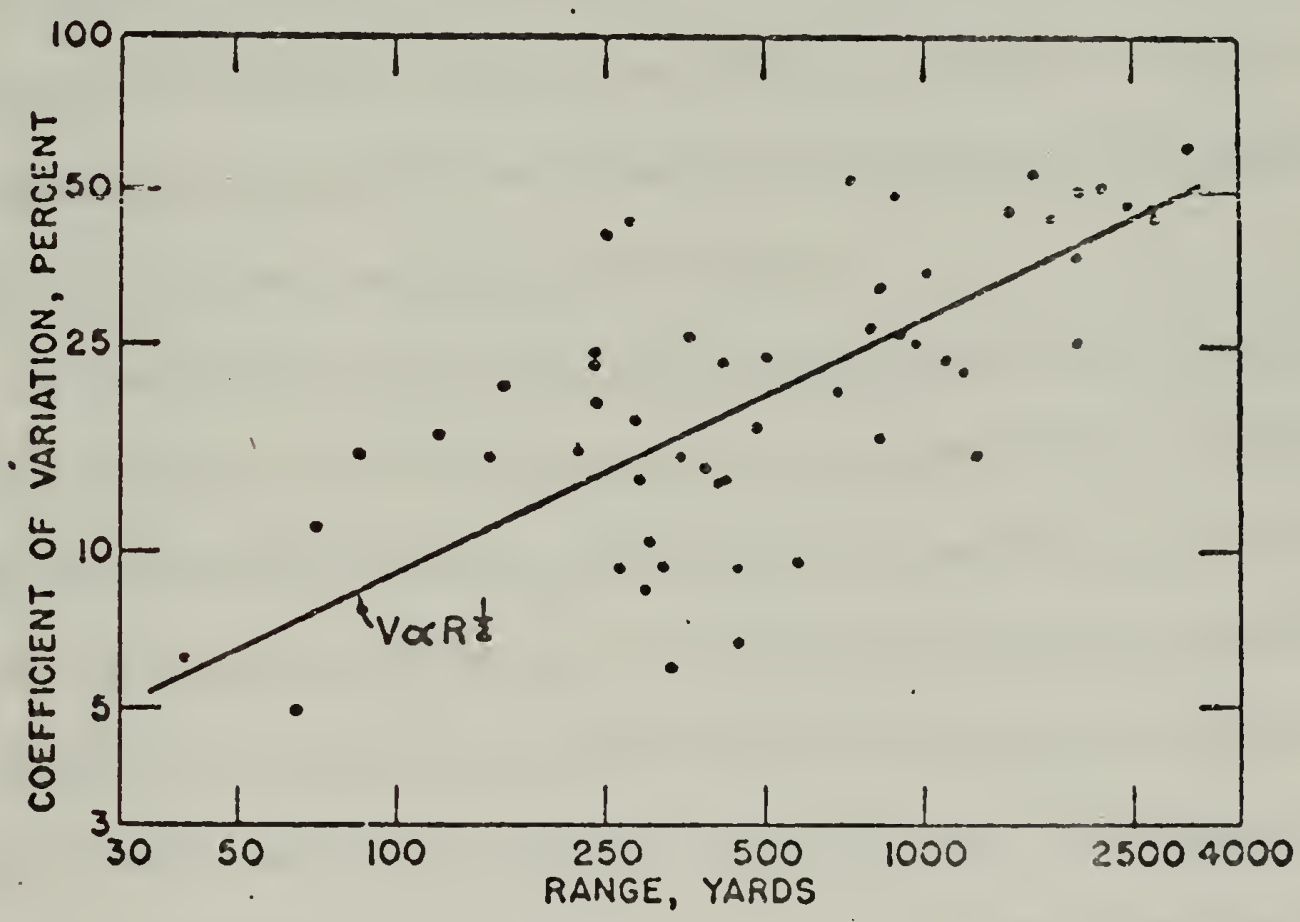

Figure 1. Coefficients of Variation of the amplitudes of direct signals plotted against the range to the receiver. Sound frequency, $24 \mathrm{kHz}$ [after Sheehy, 1950]. 

Bergmann [1946] using the application of ray optics to investigate the propagation of radiation in a medium with random inhomogeneities, derived an approximate formula which showed that the signal level fluctuations increased in proportion to the threeshalves power of the range. He pointed out the variance with Sheehy's results, but offered no explariation for the differences.

Liebermann [1951], using an autocorrelation function calculated from measured horizontal small-scale temperature inhomogeneities, showed that the refractive effects of the horizontal inhomogeneities produce a local warping (focusing or defocusing of sound) that causes the acoustic intensity to fluctuate. He found that refraction should only occur if the frequency is high enough to make the dimensions of the inhomogeneities greatly exceed the wave length of the radiation.

Mintzer [1953a] derived a first order approximation for the acoustic pressure amplitude variation of an acoustic pulse traveling in a medium with small random variations in the refractive index that are a result of random temperature variations. Assuming that the pressure amplitude fluctuations are small enough to allow the use of a single scattering approximation and that the refractive index at a point only changes during the time between pulses and not while the pulse is passing the point, he solved the Born approximation to the wave equation for the pressure amplitude of the acoustic pulse. Then using the Coefficient of Variation, $\mathrm{V}$, defined as: 


$$
v^{2}=\frac{\left\langle|\bar{P}|^{2}\right\rangle-|\langle\bar{P}\rangle|^{2}}{\left.|\bar{P}\rangle\right|^{2}} \text {, where }
$$

$$
\begin{aligned}
& \langle\rangle=\text { ensemble average } \\
& \overline{\mathrm{P}}=\text { mean presure amplitude }
\end{aligned}
$$

and by averaging the pressure amplitude over the pulse length instead of using the maximum value of the pressure amplitude as Sheehy did with his experimental data, Mintzer's theoretical analysis yialds the following. for ranges large compared with the correlation distance:

$$
\begin{aligned}
& \mathrm{V}^{2}=2 \mathrm{k}_{0}^{2} \alpha^{2} x \int_{0}^{\infty} \mathrm{N}(\rho) \mathrm{d} \rho \text {, where } \\
& \mathrm{N}(\boldsymbol{\rho})=\text { autocorrelation function of the refraction } \\
& \quad \text { index variation taken along the line between } \\
& \quad \text { source and receiver } \\
& \mathrm{k}_{\mathrm{o}}=\quad \text { wave number of the sound } \\
& x=\quad \text { range from source to receiver } \\
& \rho=\quad \text { rms refractive index variation }
\end{aligned}
$$

Using a Gaussian correlation function the coefficient of variation becomes:

$$
\begin{aligned}
& V=\left(\begin{array}{llll}
\pi^{\frac{3}{2}} & k_{0}^{2} & \alpha^{2} a
\end{array}\right)^{\frac{1}{2}} x^{\frac{1}{2}} \text {, where } \mathrm{a}=\text { correlation } \\
& \text { length }
\end{aligned}
$$



This theoretical result is in agreement with Sheehy's experimental results [Figure 1].

Later Mintzer [1953b] determined over what range the first assumption (use of a single scatterer approximation) was valid. He found that for large ranges compared with the acoustic wave length and the inhomogeneity size, and a short pulse length compared to the time required for a refractive index change:

(1) The Coefficient of Variation of the pressure amplitude is:

$$
\begin{aligned}
& \mathrm{V}^{2}=\pi^{\frac{1}{2}} \mathrm{k}_{0}^{2} \alpha^{2} \text { ar, } \mathrm{ka} \gg 1 \\
& \mathrm{~V}^{2}=\pi^{\frac{3}{2}} \mathrm{k}_{0}^{3} \alpha^{\bar{L}} \mathrm{a}^{2} \mathrm{r}, \mathrm{ka} \ll 1
\end{aligned}
$$

(2) The single-scattering assumption used is valid for:

$$
k_{0} r<<\left(k_{0} a \alpha^{2}\right)^{\frac{1}{2}}
$$

Mintzer also made a comparison with ray theory and found that for a frequency high enough to satisfy:

$$
r / k_{o} a^{2}<<1
$$

the intensity coefficient of variation computed by wave theory and ray theory are the same. 
In a third paper, Mintzer [1954] considered the second basic assumption, that the refraction index varies only during the time between pulses. He found that "if the pulse length is much less than the time constant of the refractive index correlation function, the coefficient of variation is independent of the pulse length, and the pulse-correlation function is found to be identical with the time-dependent part of the refractive index correlation function. 13

Skudrzyk [ 1957] discussed the pressure amplitude fluctuations caused by water inhomogeneities considered to be spherical "patches" that are always large in comparison with the acoustic waveiength. He showed that in the vicinity of the receiver pressure amplitude fluctuations are caused by focusing or defocusing from the lens action of the patches, and beyond a certain range the fluctuations are caused only by an interference field which results from deflected rays arriving at a point with phase shifts due to unequal paths of travel. He also pointed out that the interference component of scattering is independent of the shape of the patches, while the focusing component can be significantly affected by the shape. For example, a spheroidal patch having a vertical axis less than the horizontal axis will have a larger focusing component than a spherical patch. At low frequencies (below $20 \mathrm{kHz}$ ) interference scattering dominates, while at

3 Mintzer, D., "Wave Propagation in a Randomly Inhomogeneous Medium. III," I. Acous. Soc. Am., v. 26, p. 186, March 1954. 
higher frequencies the interference component may be so small in comparison with the focusing component that at ranges greater than the focusing range the Coefficient of Variation is almost constant. The focusing range $\left(r_{0}\right)$ is the range beyond which the focusing does not change and additional pressure arnplitude fluctuations are caused by interference only. Skudrzyk found the focusing range to be a function of frequency and path size.

$$
r_{0}=k a^{2} \text {, where }
$$$$
\mathrm{k}=\text { acoustic wave number }
$$$$
a=\text { effective radius of patch }
$$

This result is pictured graphically in Figure 2. If. the sonar frequency and the patch size are known, then the focusing range can be determined. (For example, a $24 \mathrm{kHz}$ sonar has a focusing range of 200 meters, if the patch size is assumed to be 140 centimeters).

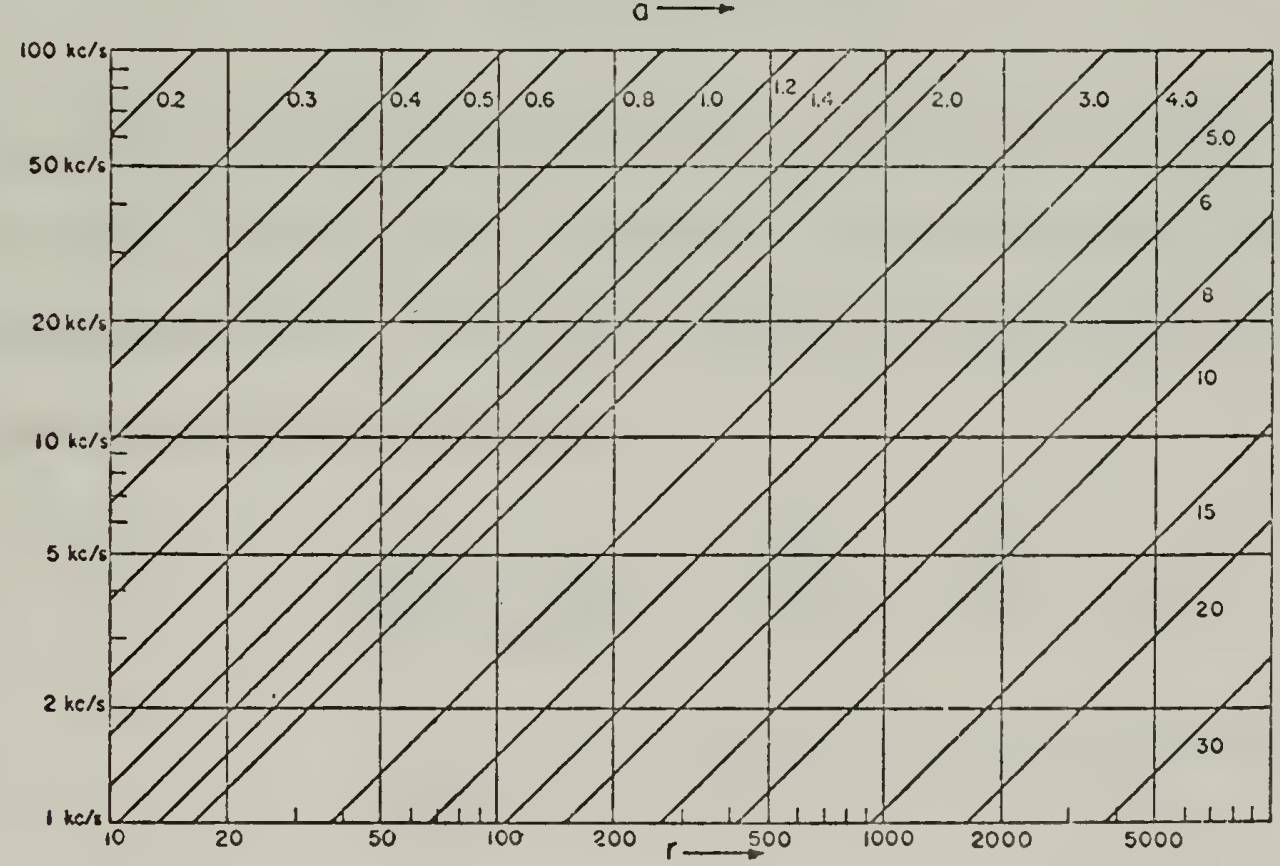

Figure 2. Focusing Range (meters) as a Functic l of Frequency $(\mathrm{kHz})$ and Patch Size (meters) [Skudrzyk, 1957]. 
Skudrzyk shows that the Coefficient of Variation of the pressure amplitude due to the focusing effect, $v_{f}$, is:

$$
V_{f}^{2}=\frac{\alpha^{2}}{2}\left(\frac{r}{a}\right)^{3} \begin{aligned}
& \text { where } \alpha \text { is the relative root-mean- } \\
& \text { square deviation of sound velocity }
\end{aligned}
$$

while the Coefficient of Variation due to the interference effect is:

$$
v_{i}^{2}=\frac{\pi}{2}^{\frac{3}{2}} k^{2} \alpha^{2} a\left(r-r_{0}\right)
$$

Beyond the focusing range, scattering is due to both focusing and interference so the Coefficient of Variation becomes:

$$
v_{p}^{2}=v_{f}^{2}+v_{i}^{2}
$$

and for spherical patches:

$$
v_{p}^{2}=\alpha^{2} \frac{\sqrt{\pi}}{2} k^{2} \text { a }\left(r-0.44 r_{0}\right)
$$

which is Mintzer's [1953b] result except for a factor of $1 / 2$. If the patches are spheroidal rather than spherical, then the focusing may be increased significantly. Whitmarsh, Skudrzyk, and Urick [1957] four.d the focusing to increase by a factor

$$
\frac{\mathrm{v}_{\mathrm{p}}(\text { Spheroidal })}{\left.\mathrm{v}_{\mathrm{p}} \text { (Spherical }\right)}=\frac{\frac{4}{\mathrm{x}^{2}}+\frac{6}{\mathrm{x}^{4}}+6}{16}
$$

$\mathrm{X}$ is the ratio of vertical to horizontal axis of the patch 

Figure 3 depicts this ratio graphically. The interference component of scattering is virtually independent of the vertical axis dimension.

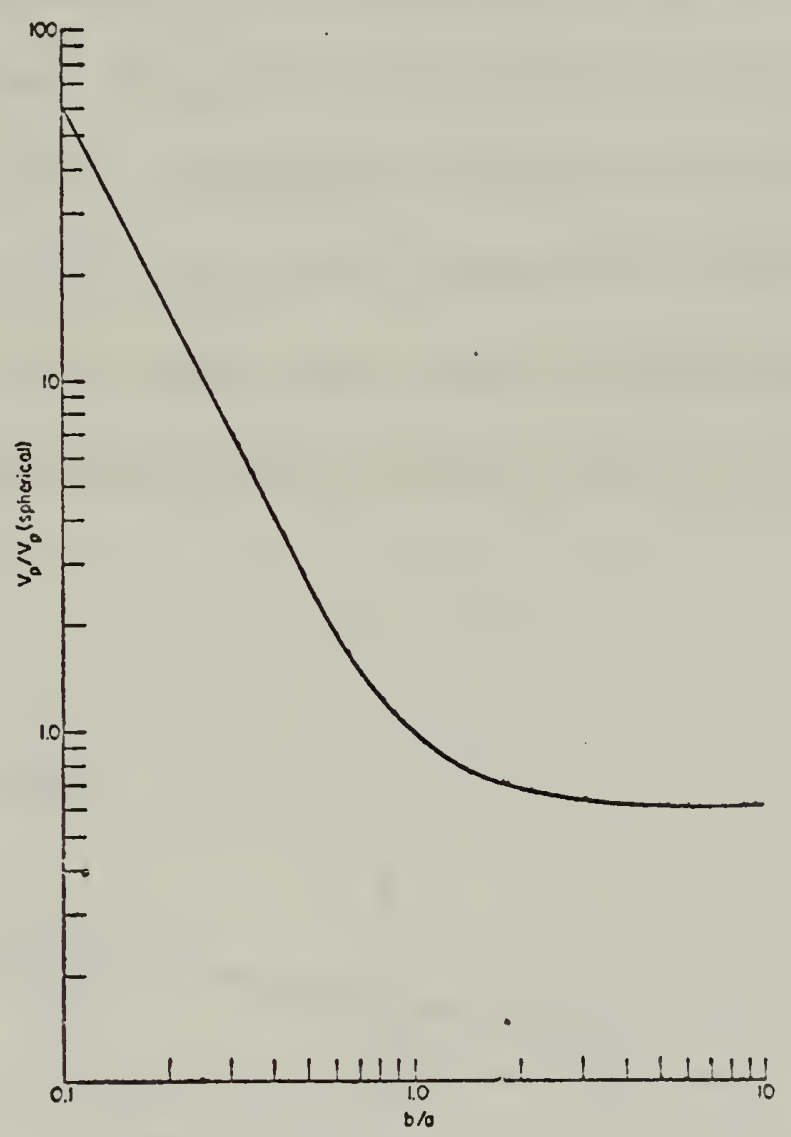

Figure 3. Increase in focusing due to spheroidal shape of a patch as a function of the ratio of the lengths of the vertical (b) to horizontal (a) axis [Whitmarsh, Skudrzyk, and Urick, 1957].

Since published experimental results pertained only to one frequency and did not include temperature anả acoustical fluctuation measurements made as one observation, Whitmarsh, Skudrzyk, and Urick [1957] conducted a series of experiments using $25 \mathrm{kHz}$ and $60 \mathrm{kHz}$ transmissions to provide additional data to test the existing theories for scattering of acoustical 

energy in the sea. They used a gradiometer bar susperded from a buoy (a 12 foot long bar with 9 thermistor beads) to record temperature gradients as small as $0.001^{\circ} \mathrm{C}$ and to record the temperature fluctuations as a function of time. They point out that all the existing theories for scattering of sound in an inhomogeneous medium are based on the assumption of a spherical patch thermal inhomogeneity (one such patch is illustrated in Figure 4). The theories further assume that the patch distribution in the medium can be represented by an exponential or Gaussian correlation function.

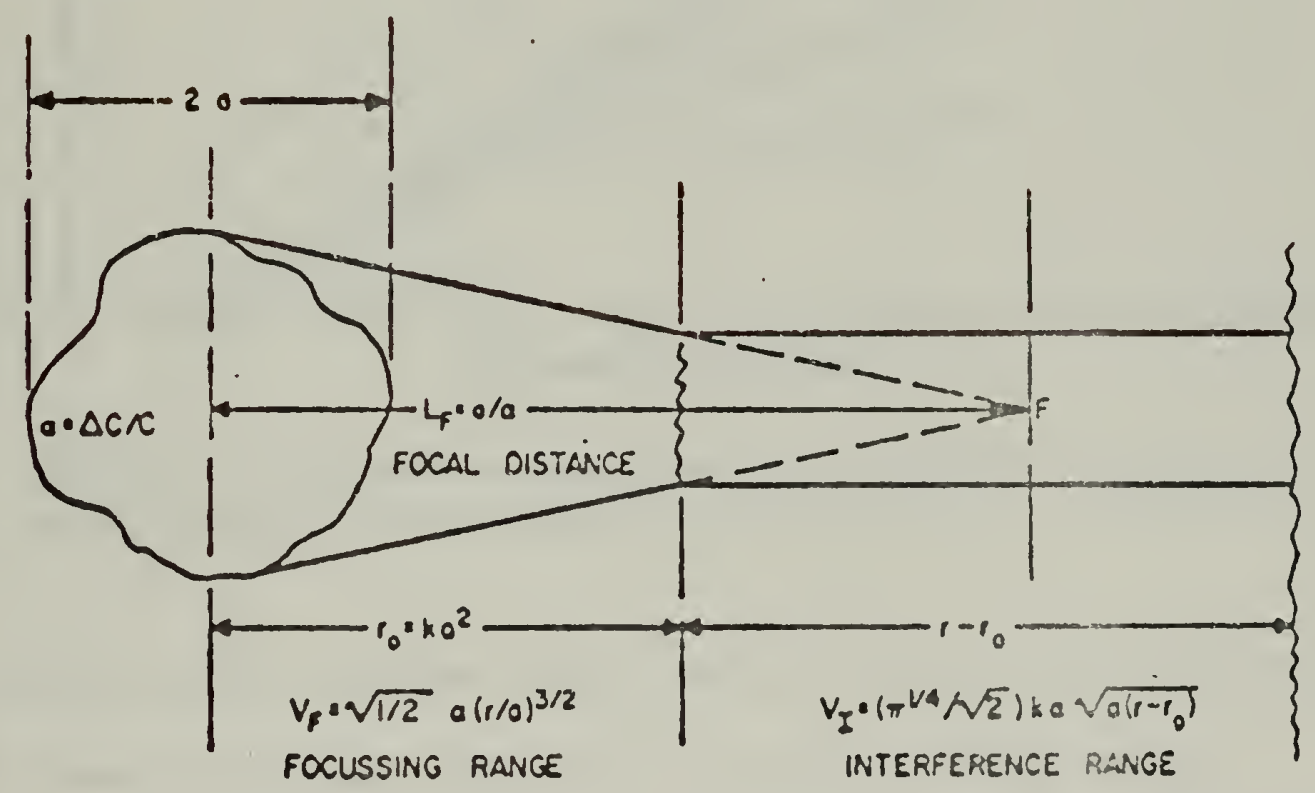

Figure 4. Illustration of the various types of acoustic scattering due to a thermal patch in the sea [Whitmarsh, Skudrzyk, and Urick, 1957].

The ternperature microstructure measurements revealed that the assumed correlation function is invalid and in fact the results sho the temperatire 

fluctuations increase with the $2 / 3$ power as the thermistor spacing increases. This is the preaiction of the Kolmogorov thecry for homogeneous turbulence, see Figure 5. However, even replacing the correlation

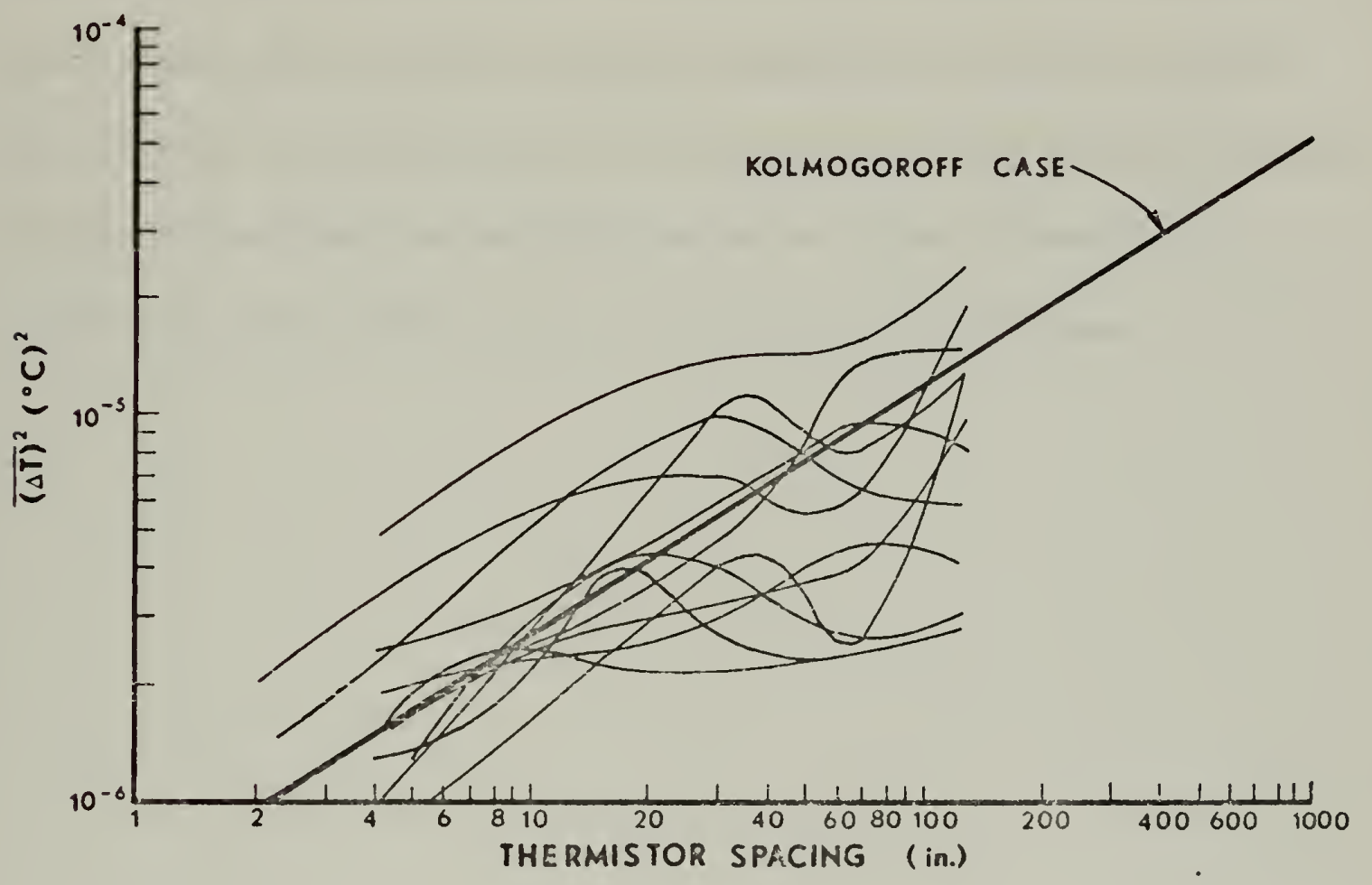

Figure 5. Horizontal temperature distributions as measured in the sea [after Whitmarsh, Skudrzyk, and Urick, 1957].

function by a new function representative of the Kolmogorov distribution, the theories for sound transmission can still be considered valid. Whitmarsh, Skudrzyk, and Urick concluded from their experiments that the present theories are capable of predicting the approximate variation in the pressure amplitude to be expected for acoustical transmissions in an inhomogeneous medium. 
Sagar [1960] analyzing the experimental data he collected off New Zealand suggests that sound intensity fluctuations which obey the Mintzer dependence on range $\left(\mathrm{V}^{2} \propto \mathrm{rf}^{2} \alpha^{2}\right.$ a) are related to insolation affects. His data is interpreted to show a diurnal cycle of growth and decay of the temperature microstructure in the surface layers of the sea with the structure most developed by late afternoon and then disappearing overnight. He also found and described the water to have a "sandwich" or layered structure rather than the classical "patch" structure. 



\section{RECENT MEASUREMENTS OF MICROSTRUCTURE}

\section{A. REVIEW}

Stommel and Fedorov [1967] used a salinity, temperature, and depth recorder (STD) to take measurements of the fine structure (nicrostructure) in the main thermocline off Timor in the Arafura Sea and Mindanao in the Philippine Sea. They were investigating reports of fine structure which began to accumulate with the advent of an instrument like the STD, which continucusly reccrds temperature and salinity as a function of depth. They found the vertical microstructure in the main thermocline to consist of hundreds of layers (laminae) of rather homogereous temperature from 2 to 40 meters thick separated by a thin layer (2 to 5 meters thick) of high temperature gradient. As an example, at one station a mean thermal gradient of $0.040^{\circ} \mathrm{C} /$ meter was found, while in a homogeneous layer the gradient was greater than $0.35^{\circ} \mathrm{C} /$ meter. By tracing some of the well defined laminae in a horizontal direction, they found the laminae extended from 2 to 20 kilometers. Though the STD resolution was only 2 meters, Stommel and Fedorov felt the microstructure existed on a smaller scale and that further investigation would be of interest.

Cooper and Stommel [1968] made microstructure measurements in the main thermocline off Bermuda using an STD. They too found a layered structure with the homogeneous layers from 3 to 5 meters thick and the 
high gradient layers between 10 and 15 meters thick. In the layers of high gradient, temperature changes as high as $0.50^{\circ} \mathrm{C}$ were found. The horizontal extent of the layers was between 400 and 1000 meters. This type structure was present out to a distance of 120 kilometers from Bermuda. Based on their measurements, Cooper and Stommel suggested that most of the Sargasso Sea exhibited the same characteristics in the main thermocline.

Woods [1968a] conducted an extensive study of the microstructure in the summer thermocline of the Mediterranean Sea. A temperature gradient meter was used to obtain measurements of temperature gradient while being lowered and temperature while being recovered. The instrument consisted of two thermistors mounted 50 centimeters apart on a vertical fiber glass rod. Only one of the thermistors was used for the temperature measurements. The instrument had an $0.01^{\circ} \mathrm{C}$ accuracy and a depth limitation of 50 meters. Woods found layers with a moderate temperature gradient that were thick in comparison with alternate layers (sheets) of high temperature gradient. Figure 6 is a plot of his results for one sounding. High gradient sheets can be seen at depths of 10,19, $24,32,41$, and 50 meters. By injecting dye from suspended packets into the different layers, Woods was able to associate laminar flow to thin high gradient layers and a weak turbulent flow to the thick homogeneous layers. The results of these two different type flows is a strong sheer across the high gradient sheets. 


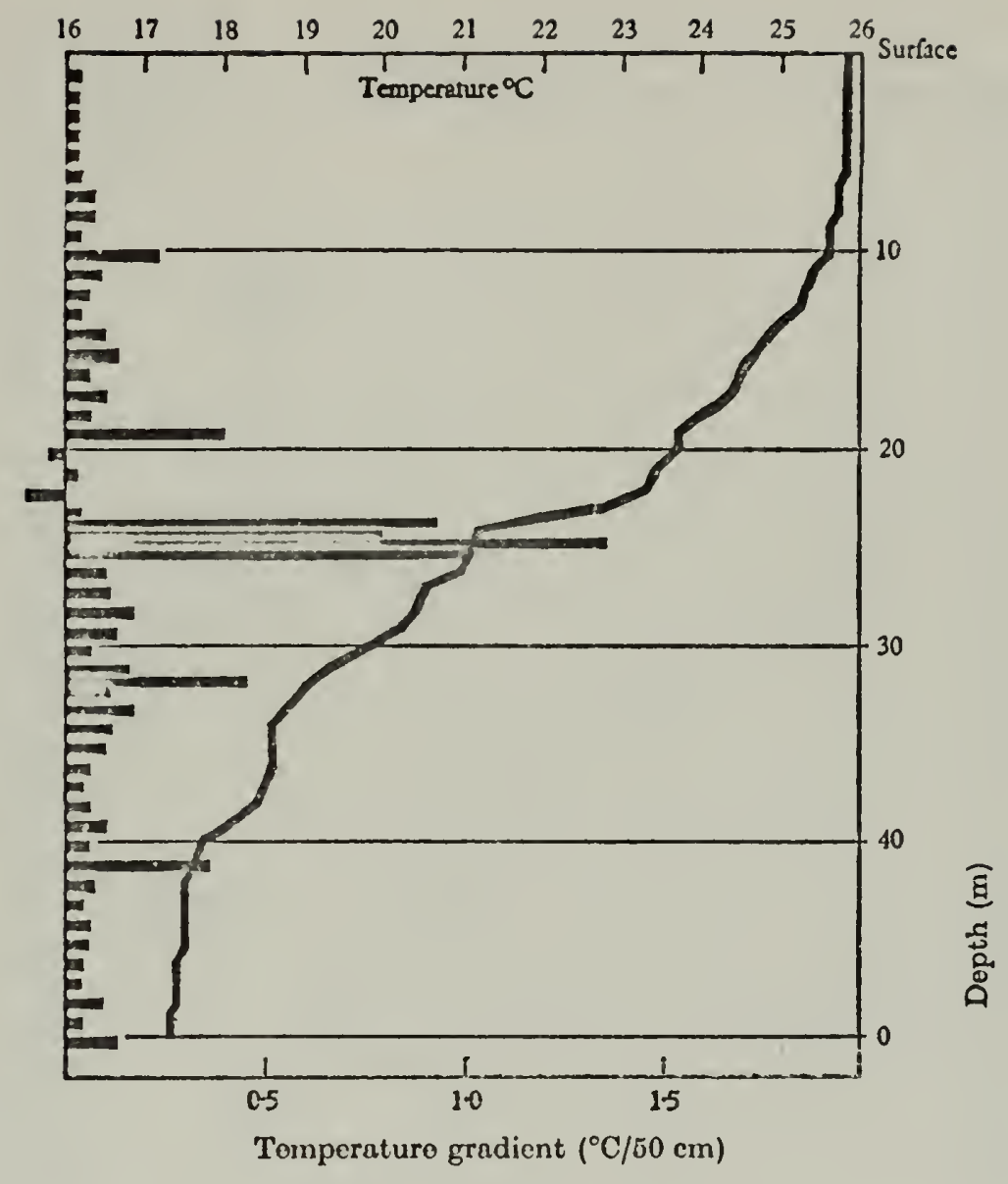

Figure 6. Temperature and temperature-gradient sounding for 063024 August 1966 in the Mediterranean Sea [Woods, 1968a].

The next year Woods [1968b] conducted another investigation of vertical heat flow through the upper ocean in the Mediterranean Sea off Malta. Again the sheets in the thermocline were found as can be seen in Figure 7. 



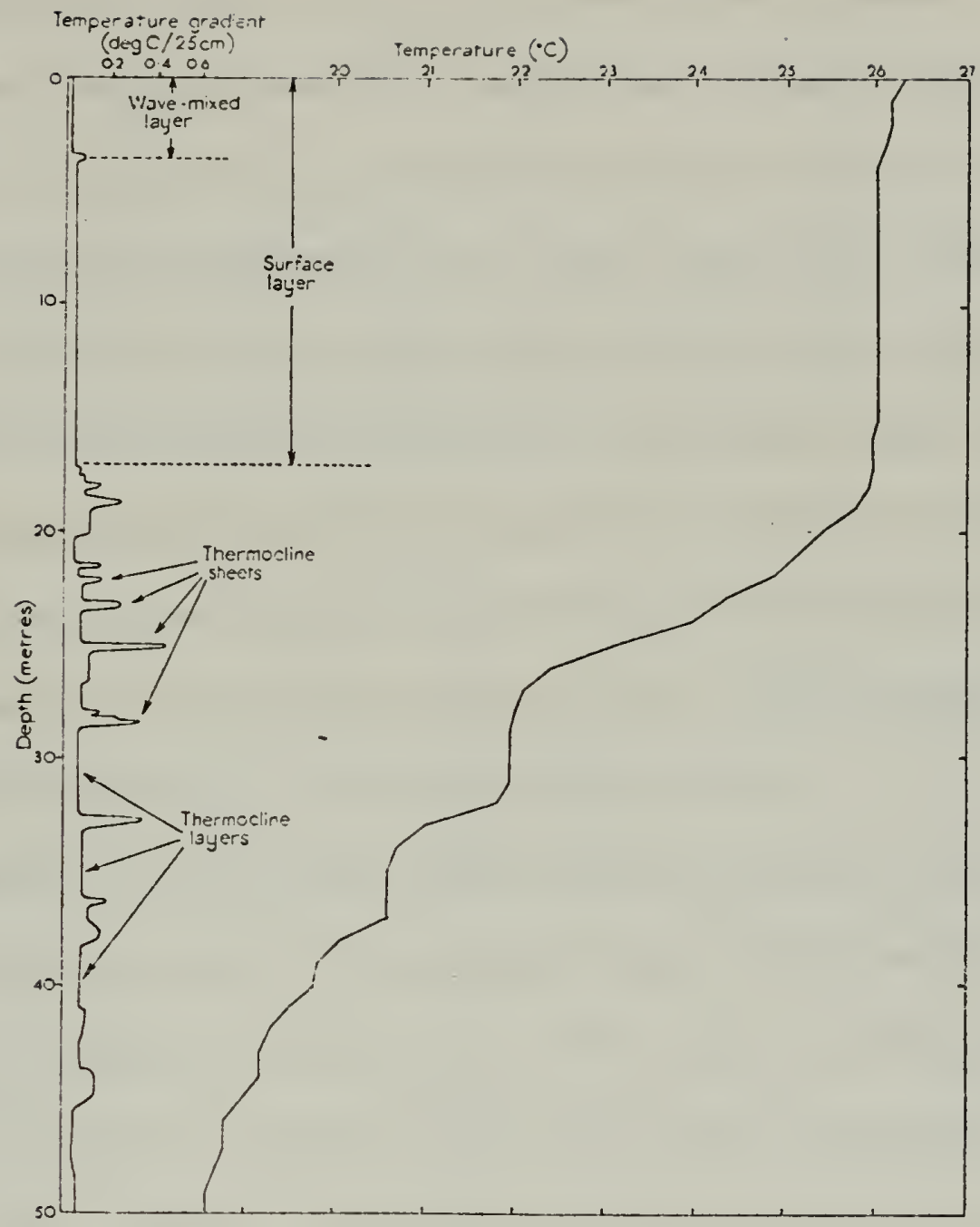

Figure 7. Temperature and temperature-gradient soundings for Maltese waters, 14 September 1967 [Woods, 1968b].

Lovett [1968] took temperature profiles in the California current off Baja California using a sound, velocity, temperature, and pressure recorder (SVTP). He too found a definite layered structure in all the temperature profiles. He suggests that the sharp gradients found in the step-like structure may have an effect on acoustic scattering, especially so at high frequencies. 

Osborn [1969] using a free fall instrument called a thermal gradiometer made microstructure measurements in the San Diego Trough, Florida current and in the central Pacific between San Diego and Hawaii. He found regions of high gradient to be about 1 meter thick and have a gradient around 10 times the mean gradient. Figure 8 is a record of the results he obtained with the gradiometer. By tracing a region of high gradient horizontally he found they extended up to 750 meters if the region is in the seasonal thermocline, but only a few hundred meters if the region is at a depth greater than 400 meters. Osborn suggests that the microstructure is on a scale of centimeter order.

Neshyba, Neal, and Denner [1969] made measurements of the temperature mirrosiructure in the Arctic Ccean using an XBT lowered mechanically from Fletcher's Ice Island (T-3). The microstructure found had a step-like structure with layers that were extremely homogeneous as can be seen in Figure 9. The transistion layer was found to be less than 20 centimeters thick. Figure $9 b$ shows a section of the trace recorded at high gain (the lip at the end of a transition layer is dive to recorder overshoot). The steps from 220 to 300 meters were found to be very stable. Figure 10 is a time series taken with the XBT modified to fall at $7 \mathrm{ft} / \mathrm{sec}$ instead of the normal $20 \mathrm{ft} / \mathrm{sec}$, and demonstrates the stability. Below 350 meters, the homogeneous layers are larger, but still are separated by steps of temperature change. This is also apparent from Figure 10. It should be noted that these XBT recordings are independent of ship motion since the ice island is a very stable platform. 



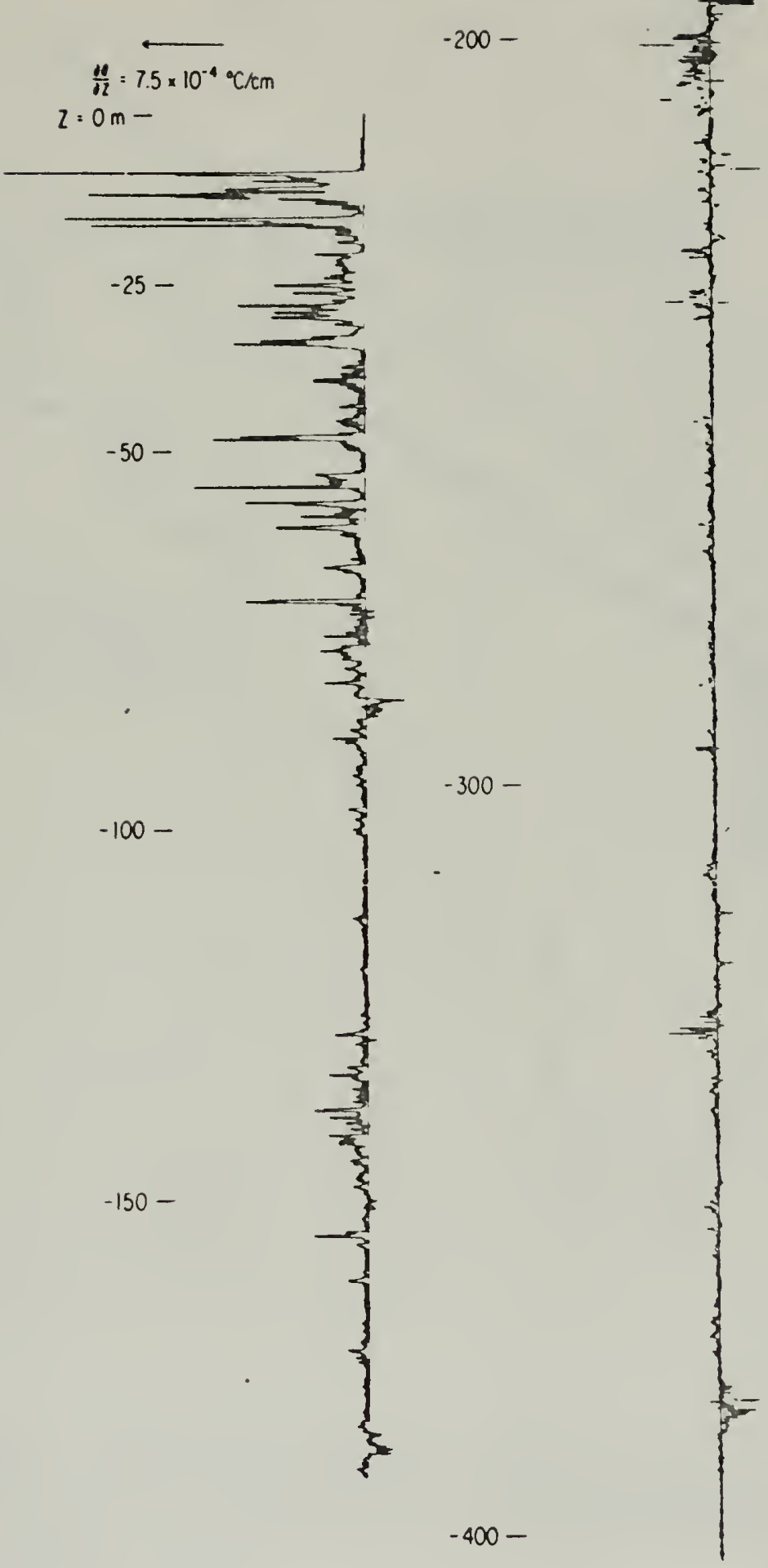

Figure 8. Corrected data from the upper poxtion of the water column in the San Diego Trough on 18 September 1968. The curve on the right is the continuation of the curve on the left [Osborn, 1969]. 



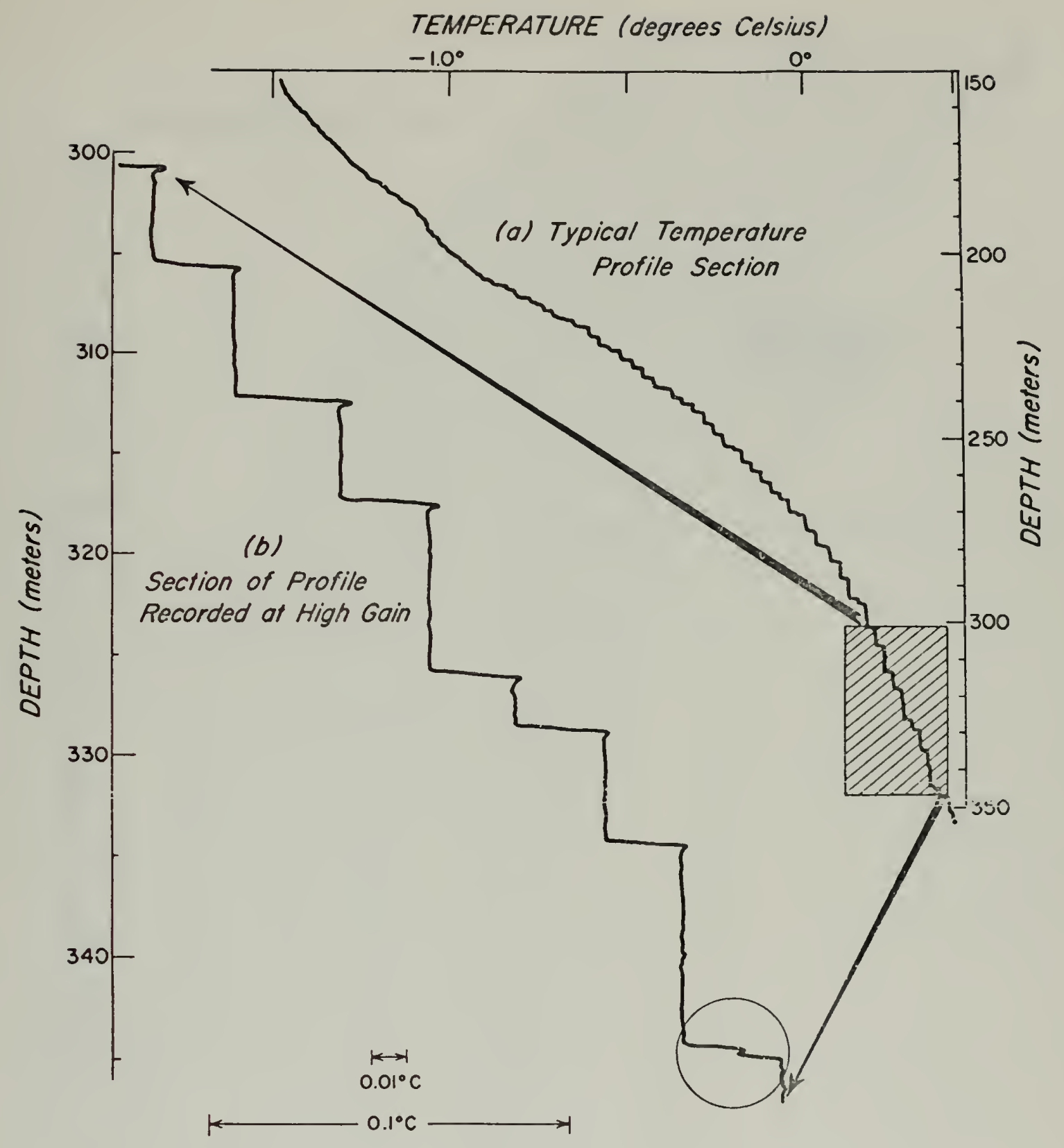

Figure 9. Vertical profile of temperature under $\mathrm{T}-3$ $8438^{\prime} \mathrm{N}, 128$ 21. 6'W, 19 Maxch 1969). Shaded section of profile (a) shown as observed in profile (b) to left [Neshyba, Neal, and Denner, 1969]. 



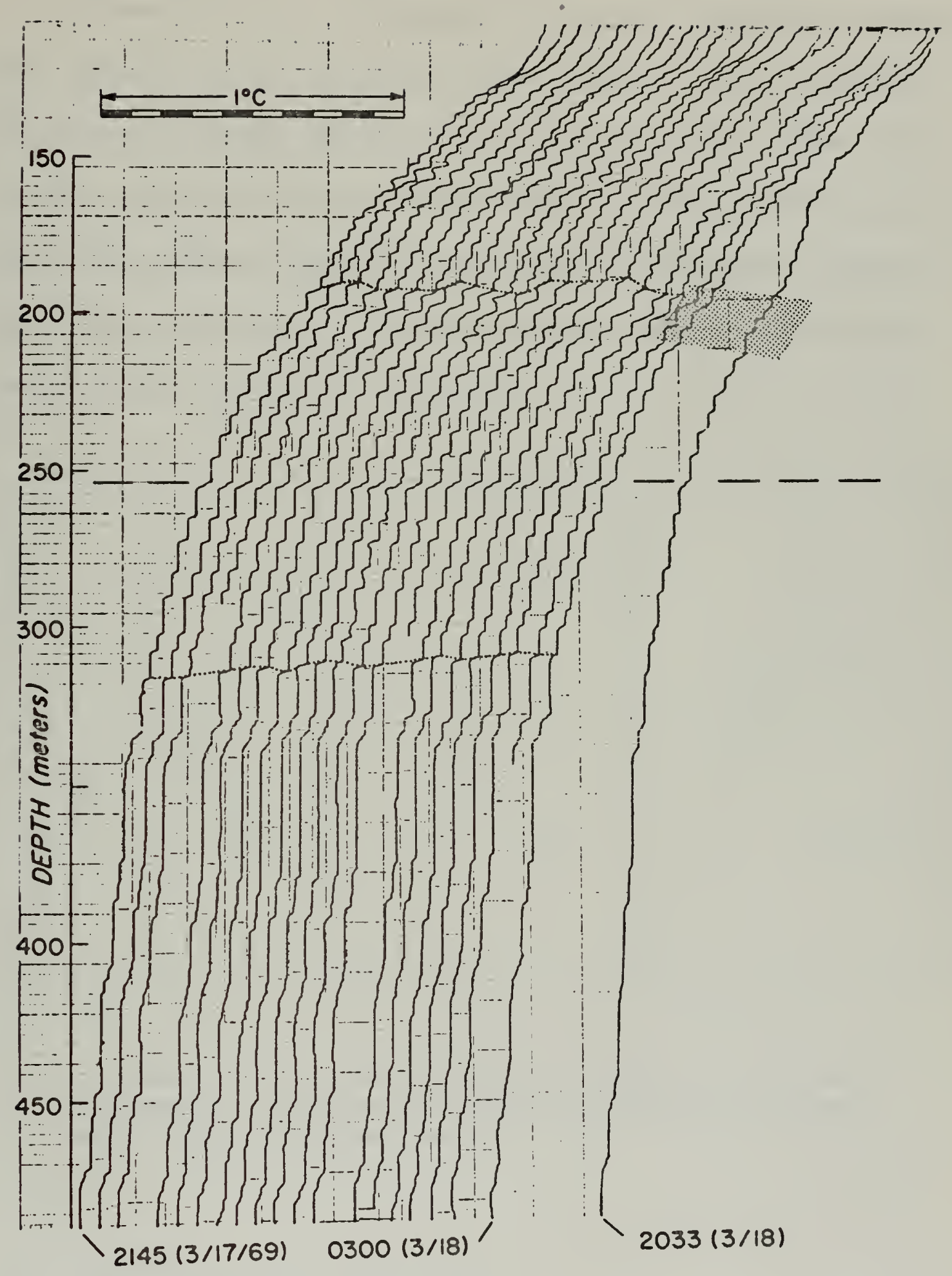

Figure 10. Time series taken from T-3 between $2145 \mathrm{Z}$ 17 March and 2033Z 18 March 1969. Temperature increases from right to left. [Neshyba, Neal, and Denner, 1969]. 

Simpson [1971], using a Bathysonde CTD system, examined the density and thermal microstructure in the western Irish Sea. He found vertical layers up to 10 meters thick and in cases, less than 1 meter thick. The average gradient was as low as $0.002^{\circ} \mathrm{C} /$ meter in layers up to 10 meters thick and as high as $2^{\circ} \mathrm{C} /$ meter in the layers of sharp gradient. Figure 11 gives three temperature profiles of the region. Horizontally the layers extended up to a distance of 3 kilometers.

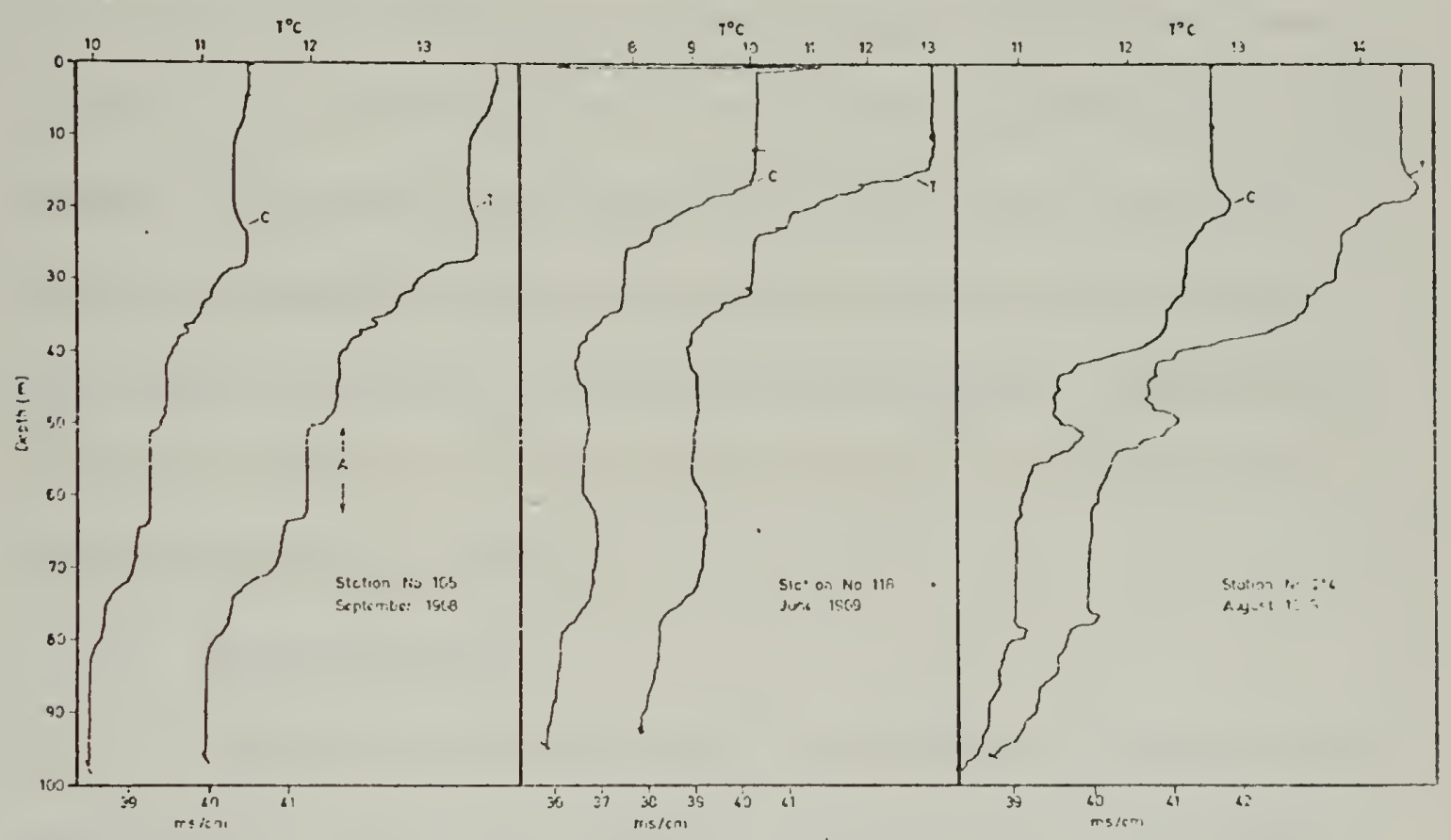

Figure 11. Unretouched Bathsonde profiles from the western Irish Sea of temperature and conductivity showing pronounced microstructure [Simpson, 1971].

\section{B. MICROSTRUCTURE MEC.HANISMS}

The exact nature of the processes producing, maintaining, or destroying the thermal microstructure still are unclear because of the complexity 

of the problem. Some mechanisms have been proposed to explain the results of observations and experiments.

\section{Insolation}

Insolation (radiation from the sun and atmosphere) results in the heating of the water's surface. The heat is transported downward mainly by mixing caused by wave action. The wind-wave action tends to eliminate the surface layer microstructure. However, Sagar [1960] suggests that after sunrise the insolation may be large enough to dominate over wave action, and so a layered structure could be transported down the thermal gradient. The depth that the layers reach is dependent on wave action. Waves are required to produce the mixing, but if they are too strong, then vigorous mixing will eliminate the layered structure. Sagar [1960] found that if cloud cover existed or if it was during a period of darkness, the microstructure was absent.

\section{Double Diffusion}

This mechanism results from the differences in the molecular diffusivity of temperature and salinity which are related by an approximate 100:1 ratio. Due to the differing diffusivity in regions where a warm saline mass of water overlies a cooler mass, the masses become unstable, forming a layered structure. Much evidence from ocean observations [Cooper and Stomme1, 1968, and Tait and Howe, 1968], a theoretical study [Turner, 1968], and laboratory experiments [Turner and Stomme1, 1964, Turner, 1967, and Stern, 1969] support this mechanism. 

Stern [1969] feels that a turbulent mixing process is taking place in thick layers that are separated by thin discontinuity layers.

\section{Internal Waves}

Woods [1968a] observed internal waves along an individual microstructure sheet to be in long coherent trains. If these waves become unstable, they would break, giving up their energy to turbulent processes causing the sheets to be eliminated by mixing. Orlanski and Bryan [1969] have proposed that internal waves breaking could also establish a step structure by leaving a "blob" of water behind that would spread out into a thin layer.

\section{Current Shear}

Current shear can cause vertical turbulent mixing when the Richardson Number is less than 0.25 [Niles and Howard, 1963]. J'his turbulence occurs as a patch which is smeared out, leaving a scar that can lead to microstructure.

Lovett [1969] and Pingre [1969] have proposed that these isolated turbulent patches can also be spread horizontally by turbulence and thus form a sheet. Dye studies illustrate this layering in the deep ocean [Schuert, 1969].

The complexity of the problem is apparent when it is realized that not one, but probably a combination of these mechanisms is occurring simultaneously. 


\section{INFORMATION CONCERNING THE THERMAL MICROSTRUCTURE THAT CAN BE GATHERED FROIM EXISTING EXPENDABLE BATHYTHERMOGRAPH (XBT) RECORDS}

\section{A. AVAILABLE DATA}

Introduction of the XBT system provided a method relatively inexpensive, yet at the same time accurate, of obtaining temperaturedepth profiles originally developed for ASW operations, the system's application has been expanded to include Navy research ships and merchant ships equipped under the Eleet Numerical Weather Central (ENWC), Cooperative Oceanographic Observation Prcgram. The commercial ship routes of the Pacific and Atlartic Occans dre a principal source of ocean temperature structure data. XBT's taken during a cruise are forwarded at the end of the cruise to FNWC, Monterey for digitizing and then archived for further use. One copy of the digitized record along with the original XBT traces is sent to the National Oceanographic Data Center for permanent storage in their archives. As of May 1971, approximately 60,000 XBT's had been digitized by FNWC, Monterey and some 28,000 of these were from the northern Pacific area [Dale, 1971].

\section{B. EXPENDABLE BATHYTHERMOGRAPH SYSTEM}

The expendable bathythermograph system currently in use is manufactured by the Sippican Corporation of Marion, Massachusetts. The system is composed of the probe (the expendable part of the system), 

a launcher, and a recorder. It is an automatic system for measuring and recording sea temperature as a function of depth. The specifications for the system are given in Table I [Sippican Corporation, 1968]:

\section{TABLE I}

Temperature range

Temperature accuracy

Depth range

Depth accuracy

Deployment cycle time

Gradient resolution

Full scale stylus travel $28^{\circ} \mathrm{F}$ to $96^{\circ} \mathrm{F}$

$-2^{\circ} \mathrm{C}$ to $35^{\circ} \mathrm{C}$

$+0.4^{\circ} \mathrm{F}$

$\pm 0.2^{\circ} \mathrm{C}$

0 to 1500 feet

0 to 2500 feet

$\pm 2 \%$ or 15 feet, whichever is greater

90 seconds for 1500 ft drop

$63 \%$ of a step change in temperature in 3 feet; $95 \%$ of a step change in 9 feet

I second

The probe is released from a cannister which pays out wire to compensate for the movement of the launcher platform while the probe itself pays out wire to compensate for its fall. The probe falls with a velocity of $20 \mathrm{ft} / \mathrm{sec}$, and has been found to have an average terminal velocity of $19.9 \pm 0.7 \mathrm{ft} / \mathrm{sec}$ [Gouzie, Sanders, and Littlehale, 1966]. A thermistor is mounted in the nose of the probe, and as the probe descends through the water column, the water temperature is sensed 
as it flows past the thermistor and out the tail of the probe. The response time for the thermistor is 110 milliseconds nominal and 130 milliseconds maximum [Choate, 1970]. The temperature resolution of the probe is dependent on the magnitude of the temperature change over a certain depth interval.

The recorder uses a pressure sensitive paper for recording the temperature profile. The paper moves vertically at a constant rate proportional to the fall rate of the probe. The chart paper displays $311.656 \mathrm{ft} /$ in or $92.307 \mathrm{~m} /$ in and $9.714^{\circ} \mathrm{F} /$ in or $5.286^{\circ} \mathrm{C} /$ in , giving a resolution of $2 \mathrm{ft}$ and $0.1^{\circ} \mathrm{F}$ for visual redding.

\section{DIGITIZING}

The digitizing process used by FNWC, Monterey is completely described by Bauer [1969]. The CALMA Model 480 digitizer is a X - Y recorder that records at least every 0.15 inch of movement in either the $\mathrm{X}$ or $\mathrm{Y}$ direction. The speed the stylus is moved down the trace determines the number of points recorded. The maximum resolution is 0.01 inches of movement in the $\mathrm{X}$ or $\mathrm{Y}$ direction which is equivalent to $.053^{\circ} \mathrm{C}$ or $.097^{\circ} \mathrm{F}$ in temperature and 0.92 meters or 3.12 feet in depth. Each trace is digitized twice.

The digitized data is further processed using a DIGITBT computer program. The program first puts the data in a common format. Then the two digitized records are compared point by point in the $X$ and $Y$ dimensions. If the maximum differences in both directions are less 

than 0.035 inches $\left(0.186^{\circ} \mathrm{C}\right.$ or $0.340^{\circ} \mathrm{F}$ in temperature and 3.22 meters or 10.92 feet in depth), the two records are averaged on a point by point basis to produce an average trace which is used for the remainder of the DIGITBT program. If the deviation in either direction is greater than 0.035 inches, then the records are rejected and must be redigitized.

The program next selects points that will be printed out and stored on the XBT record tapes. These points are called INELECTION POINTS. Both the first and last points of the record are automatically selected as inflection points. The other points of the record are screened in "batches". For each batch the observation is found that is most different from that expected by linear interpolation between the end points of the batch. If that djfference exceeds 0.015 inches $\left(0.0790^{\circ} \mathrm{C}\right.$ or $0.146^{\circ} \mathrm{F}$ and 1.38 meters or $4.68 \mathrm{feet}$ ) then the observation is selected for output. Otherwise the batch expands to include the next value and the inflection test is repeated until an inflection is found and until the entire record has been screened. Any point that can be reconstructed by linear interpolation is not required and therefore is removed from the output. The DIGITBT program converts the inflection points from inches to meters and Centigrade degrees for output. The depth is rounded to the nearest whole unit and temperature to the nearest tenth of a degree. Figure 12 is an example output from the DIGITBT program and is the information stored in FNWC, Monterey's records. The title line contains a ship identification code, day, month, year, time, 



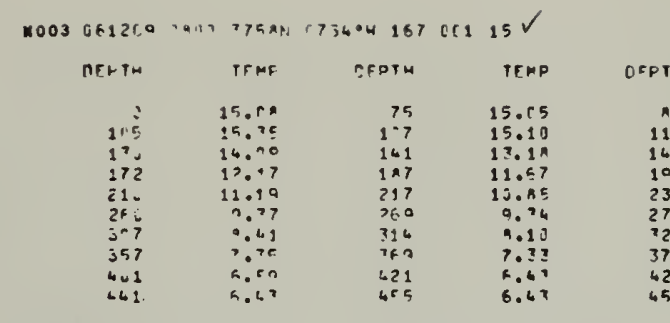

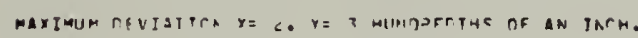

\begin{tabular}{|c|c|c|c|c|c|}
\hline PIH & TEMP & TEETH & TEMD & OEPTY & TEWP \\
\hline$A C$ & 15.61 & 17 & 15.63 & 97 & 15.53 \\
\hline 117 & 14.15 & 114 & 14.65 & 123 & 14.22 \\
\hline 164 & 13.13 & 152 & 12.63 & 160 & 12.37 \\
\hline 106 & $11.5 ?$ & 200 & 11.46 & 203 & 11.21 \\
\hline 232 & 10.53 & $23 t$ & 10.37 & $2+3$ & 1.09 \\
\hline $27 k$ & $\begin{array}{l}3.94 \\
7.95\end{array}$ & $\begin{array}{l}29 n \\
328\end{array}$ & $\begin{array}{l}0.62 \\
7.78\end{array}$ & $\begin{array}{l}302 \\
356\end{array}$ & 8.66 \\
\hline 371 & 7.20 & 387 & 7.61 & 391 & 6.63 \\
\hline 426 & 3.43 & 426 & A.61 & 431 & b.se \\
\hline 657 & 6.63 & & & & \\
\hline
\end{tabular}

Figure 12. Digitized record [Dale and Stevens, 1970 ].

latitude, longitude, base line temperature, grid type, and maximum depth of probe (15 for $1500 \mathrm{ft} \mathrm{XBT} \mathrm{or} 25$ for $2500 \mathrm{ft} \mathrm{XBT}$ ). Figure 13 is a comparison of the oxiginal $\mathrm{XBT}$ trace and the reconstructed trace from a DIGITBT record similar to the example in Eigure 12. As could be expected, the digitizing process has eliminated some of the small scale microstructure.

For this thesis the data used is from the FNWC, Monterey's data bank that has been processed using the DIGITBT program. 


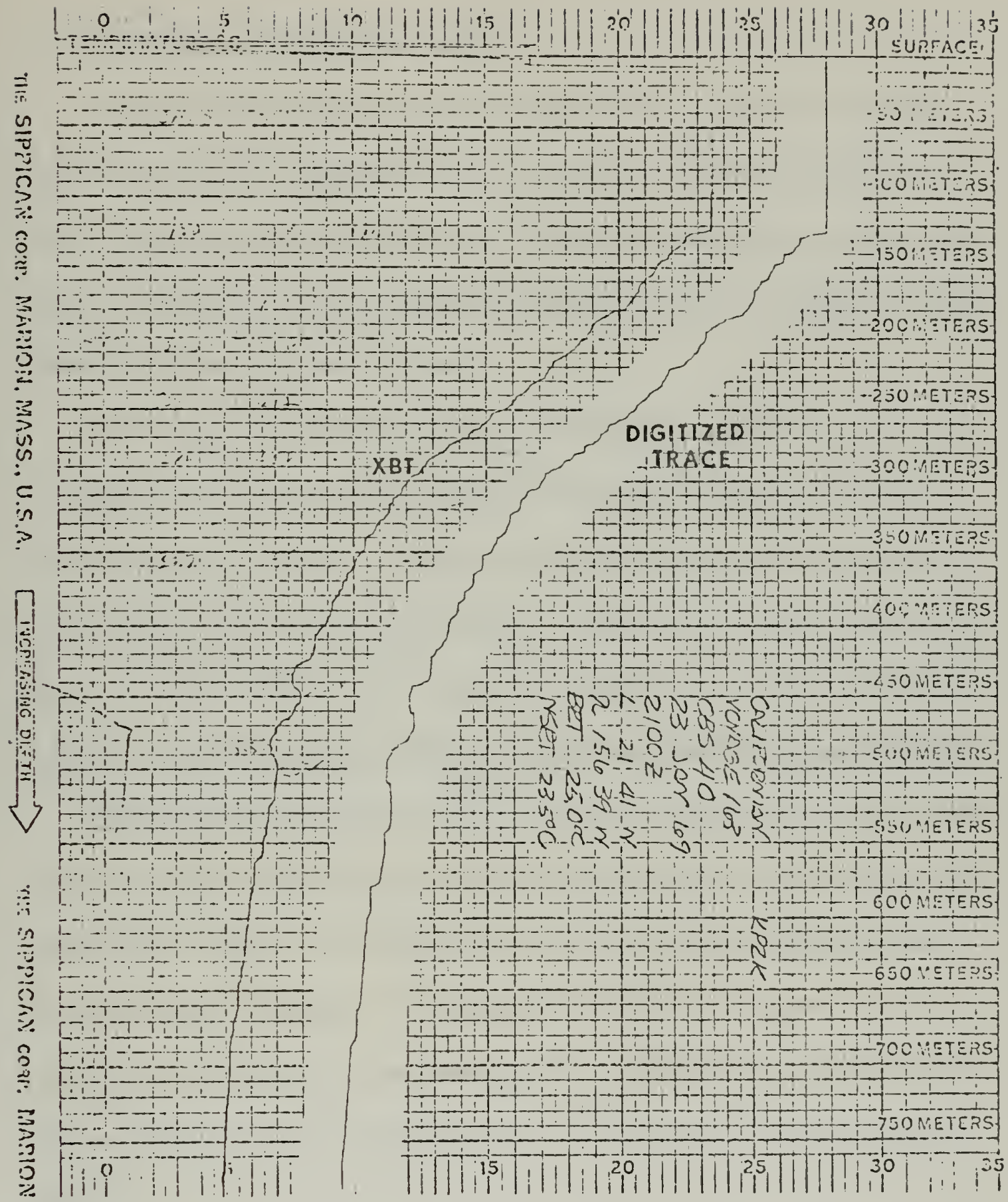

Figure 13. Comparison of an original XBT trace with the trace reconstructed from the digitized data. The digitized trace is plotted to a scale of 90 meters/in due to computer requirements while the original trace is scaled to 92.3 meters/in. 



\section{THERMAL MICROSTRUCTURE}

Thermal microstructure for the purpose of this thesis is defined as the temperature fluctuations as a function of depth that exist on a scale that is smallex than the scale of the gross temperature structure which is derived from the large scale processes such as weather, latitude, season, and water mass transport.

To analyze the XBT trace for microstructure, a method was sought to separate the temperature perturbations (microstructure) from the mean of the XBT trace. A method to filter the trace and subtract the mean portion of the curve was desired. Several type filters were considered. A binomial filter was selected primarily because Roden [1971] had applied a modified binomial filter to similar data with favorabie results.

Roden utilized a low pass filter to develop a mean series. This mean series was then subtracted from the original series to give a temperature perturbation series. The infrmation was therefore effectively passed through a high pass filter. Using this scheme the temperature pertuxbations are considered to be associated with the thermal microstructure. If the original series is denoted by $s(z)$, then the mean series, $s^{*}(z)$, is found by:

$$
s *(z)=\sum_{i=-\frac{m}{2}}^{\frac{m}{2}} w(i) s(z+1)
$$



where $w(i)$ is a smoothing array that has the following properties:

$$
\begin{aligned}
& w(i)=w(-i) \\
& \sum_{i} w(i)=1
\end{aligned}
$$

The smoothing array is found by evaluating the below modified binomial sequence.

$$
w(i)=\left[\frac{m !}{(m-i) ! i !}\right]^{p} / \sum_{i}\left[\frac{m !}{(m-i) ! j !}\right]^{p}
$$

The parameter, $m$, is even and controls the width (number of weighted points) of the array. The parameter, $\mathrm{p}$, is an arbitrary exponent which controls the peakedness of the array. Roden found that a statistically stable mean series could be obtained if $m=40$, with $p=0.5$. Increasing $\mathrm{m}$ produces more smoothing, but the series is physically shortened as will be seen later. The response of the modified filter, $r(k)$, is given by

$$
r(k)=1-\sum_{i} w^{\prime}(i j \cos (\mathrm{ik} \Delta l)
$$

where $k=$ wave number $(2 \pi /$ wave length $)$ and $\Delta \ell=$ filtering interval (distance between the 41 weighted points). Figure 14 is the solution for the response function for filtering intervals $(\Delta \ell)$ of 1,2 , and 3 meters. The smaller $\Delta l$ the sharper is the response curve at a cut-off wave length, however, the cut-off wave length is lowered. It should be remembered that with a binomial filter the amplitude response is not very predictable in the region of cut-off. 



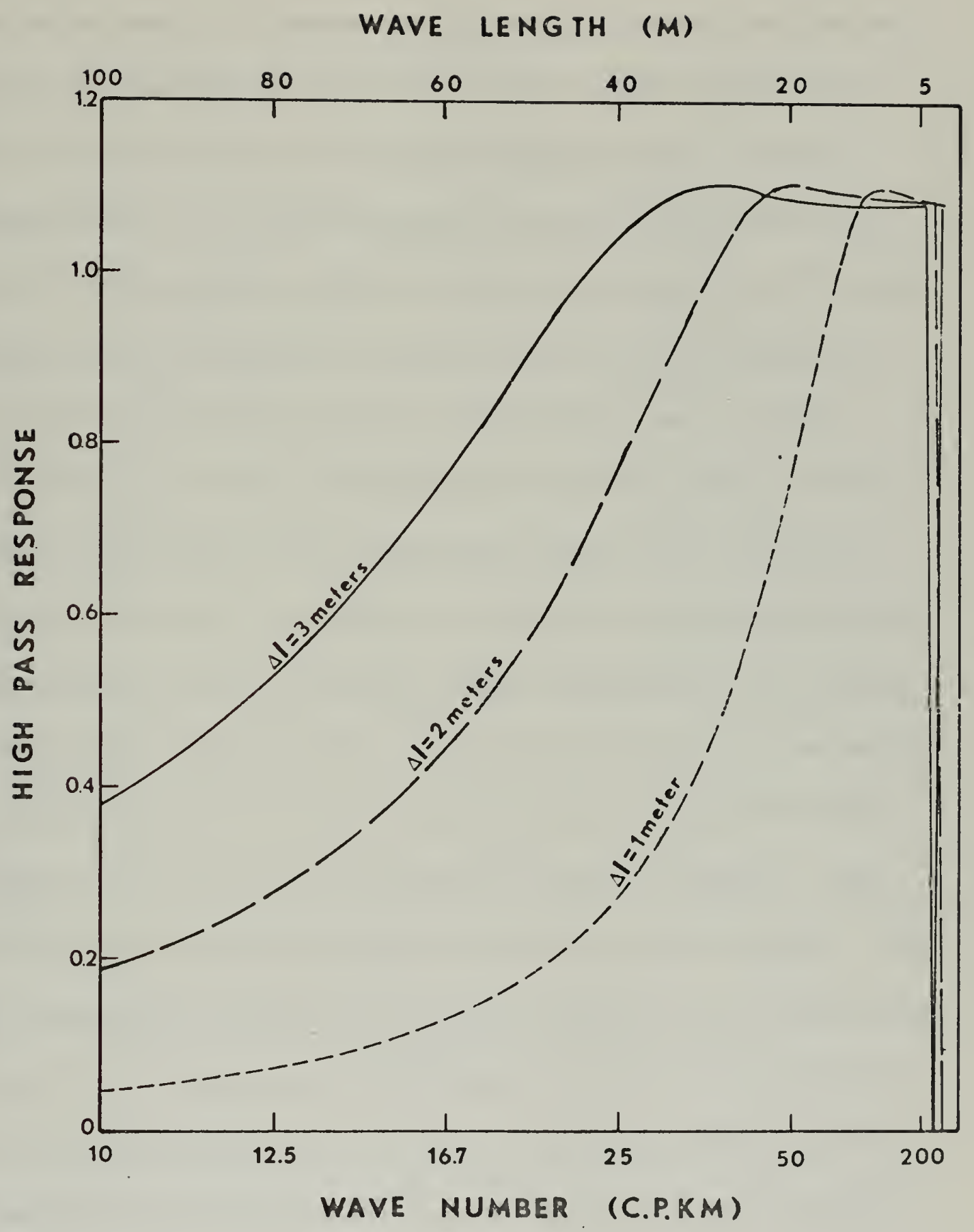

Figure 14. Computer solution for modified binomial filter with filtering intervals of 1,2 , and 3 meters. 

A theoretical XBT trace was constructed for test purposes. An isothermal layer of 50 meters was followed by a thermocline simulated with an exponential decay function $\left(\mathrm{e}^{-\alpha z}\right)$ that decayed in 650 meters. The microstructure was constructed by superimposing a sine wave function $(\sin 2 \pi \mathrm{kz})$ on the exponential function. The amplitude was varied to represent the temperature fluctuations desired, and the wave length varied to represent the microstructure layer thickness scale. Figure 15 is a composite picture of a theoretical trace, the mean filtered trace, and the resulting temperature perturbation curve for a 7 meter wave length, $0.5^{\circ} \mathrm{C}$ temperature variation, and a filtering interval of 1 meter. As expected, the perturbation curve is the input sine wave curve with both the wave length and amplitude of the temperature variation being preserved. Figure 16 is another set of theoretical curves showing that for the one meter filtering interval if the wave length is 34 meters and the temperature amplitude variation is again $0.5^{\circ} \mathrm{C}$ the temperature perturbation amplitude has been amplified, while the wave length is preserved. It should be noted that the 34 meter wave length is on the slope part of the response curve in Figure 14, and this shows the instability of the filter in this region as predicted. If the filtering interval is increased to 3 meters then the original sine wave amplitude will again be passed. 



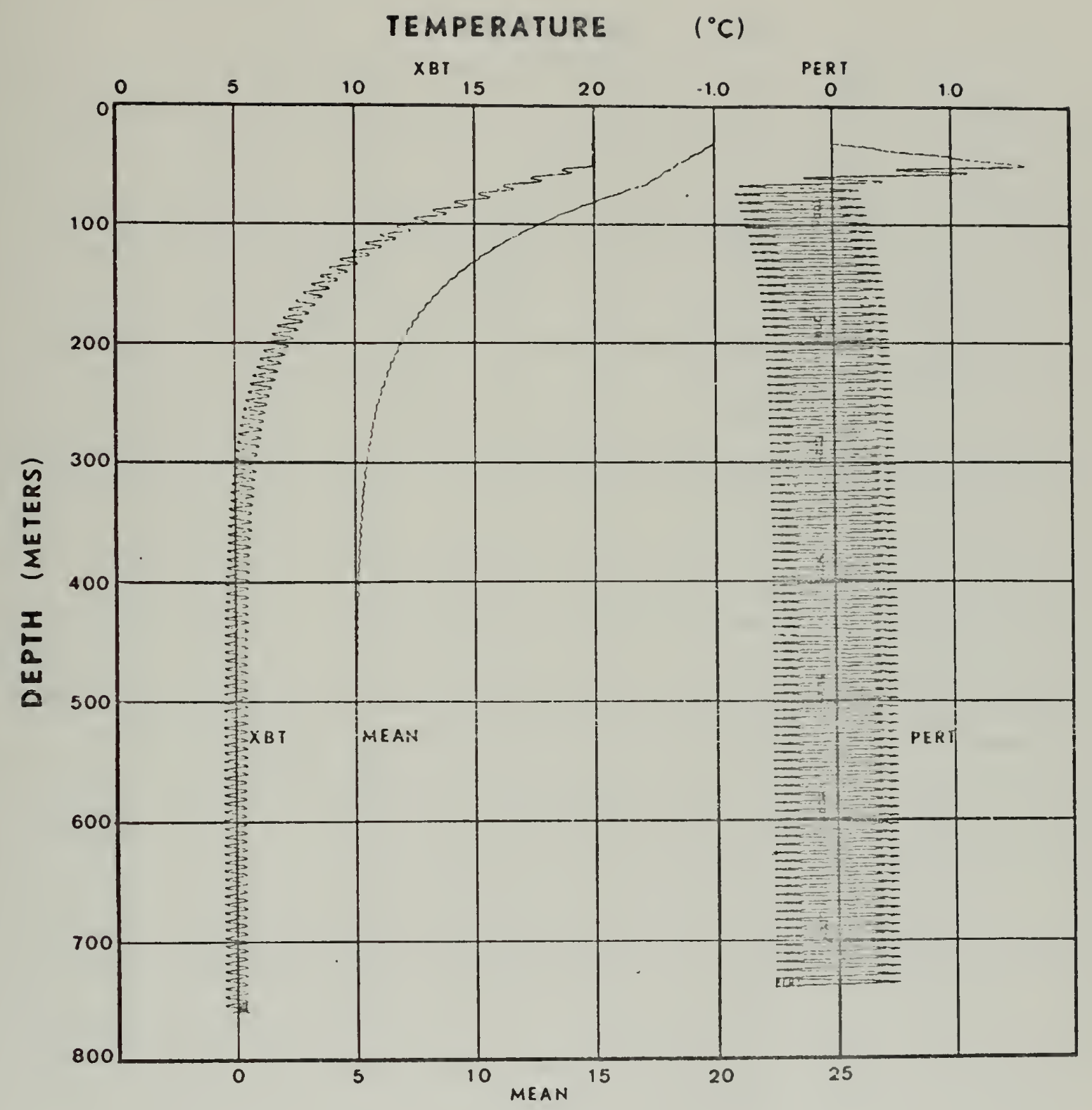

Figure 15. Result of modified binomial filter applied to Theorectical XBT trace. From left to right, Theoretical XBT trace, Mean curve, and Temperature Perturbation curve for a 7 meter wave length, $0.5^{\circ} \mathrm{C}$ Temperature variation, and a 1 meter filtering interval. 



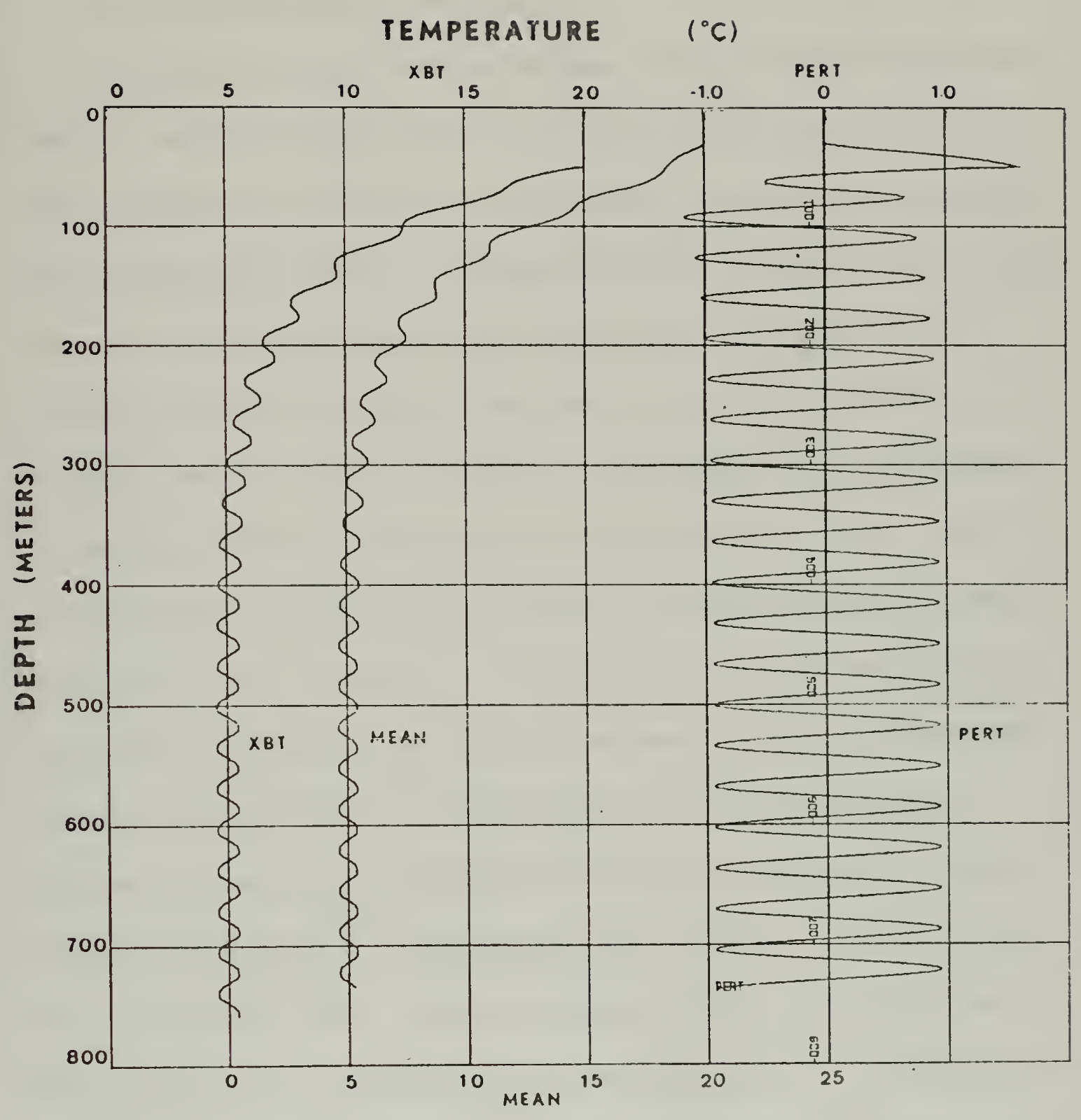

Figure 16. Result of modified binomial filter applied to theoretical XBT trace showing the amplification in the temperature perturbation amplitude when the wave length of the perturbation is on the slope part of the response curve of Figure 14. From left to right., Theoretical XBT trace, Mean curve, and Temperature Perturbation curve for a 34 meter wave length, $0.5^{\circ} \mathrm{C}$ Temperature variation, and a 1 meter filtering interval. 



\section{E. DATA SELECTED FOR ANALYSIS}

A selection of 129 XBT traces has been analyzed for this thesis using Roden's modified binomial filter with 1,2 , and 3 meter digitizing intervals. All of the traces were from the northern Pacific Ocean. Digitized information was furnished by the Fleet Numerical Weather Central (FNWC), Monterey, and was processed using the IBM $360 / 67$ at the Naval Postgraduate School's W. R. Church Computer Center. The traces were extracted from the FNWC data bank by selecting geographic grid squares. The selection of these grid squares was based on three criteria. The first and most important was to get a sampling from the Subtropic, Subarctic, Kuroshio Transport and Region of Confluence climatic regions proposed by Tully [1964]. Secondly it was desired to secure data for comparison with copies of original XBT traces obtained from J.F.T. Saur of NOAA's National Marine Fisheries Service at the Fishery-Oceanography Center in La Jolla, California. The third criteria was to request a reasonable quantity of data from FNWC. Data was desired for all months of the year in each grid square, but unfortunately the coverage fell short of expectations. Another shortcoming in the data is that the Subarctic region has not been sampled as often with XBT as the Subtropic region and therefore, data is not very plentiful from this area. 



\section{F. DESCRIPTION OF MICROSTRUCTURE ON XBT TRACE}

Figure 17 is a composite diagram of an actual XBT trace, the digitized trace, a mean curve found with Roden's filter, and the temperature perturbation curve using a I meter filtering interval. The scale of the actual XBT trace is 92.3 meters/in while the other three traces are scaled 90 meters/in for computer requirements. By comparing the actual $\mathrm{XBT}$ trace and the digitized reproduction it can be seen that some of the very fine scale temperature microstructure has been eliminated by the digitizing process and some larger scales appear to have been amplified by the process. The exactness of the digitized trace is considered to be sufficiently reliable to achieve results that are realistic and comparable.

The first and last 20 meters of the mean trace are lost because the filter used 20 points at 1 meter intervals on both sides of a depth to compute the mean value for that depth. If a 2 meter filtering interval is used, then a total of 80 meters is lost and the 3 meter interval reduces the length by 120 meters. Since the XBT record is comparatively short to begin with, a 750 meter trace would be reduced by 16 percent when using the 3 meter interval, and if the trace is a $1500 \mathrm{ft}$ XBT (460 meters) 26 percent of the record will be lost.

By closely comparing the digitized XBT trace and the temperature perturbation curve in Figure 17, it can be seen that any time an inflection point (as defined in Section IV C) is encountered on the XBT trace, a change in the slope results on the temperature perturbation curve. The number of 



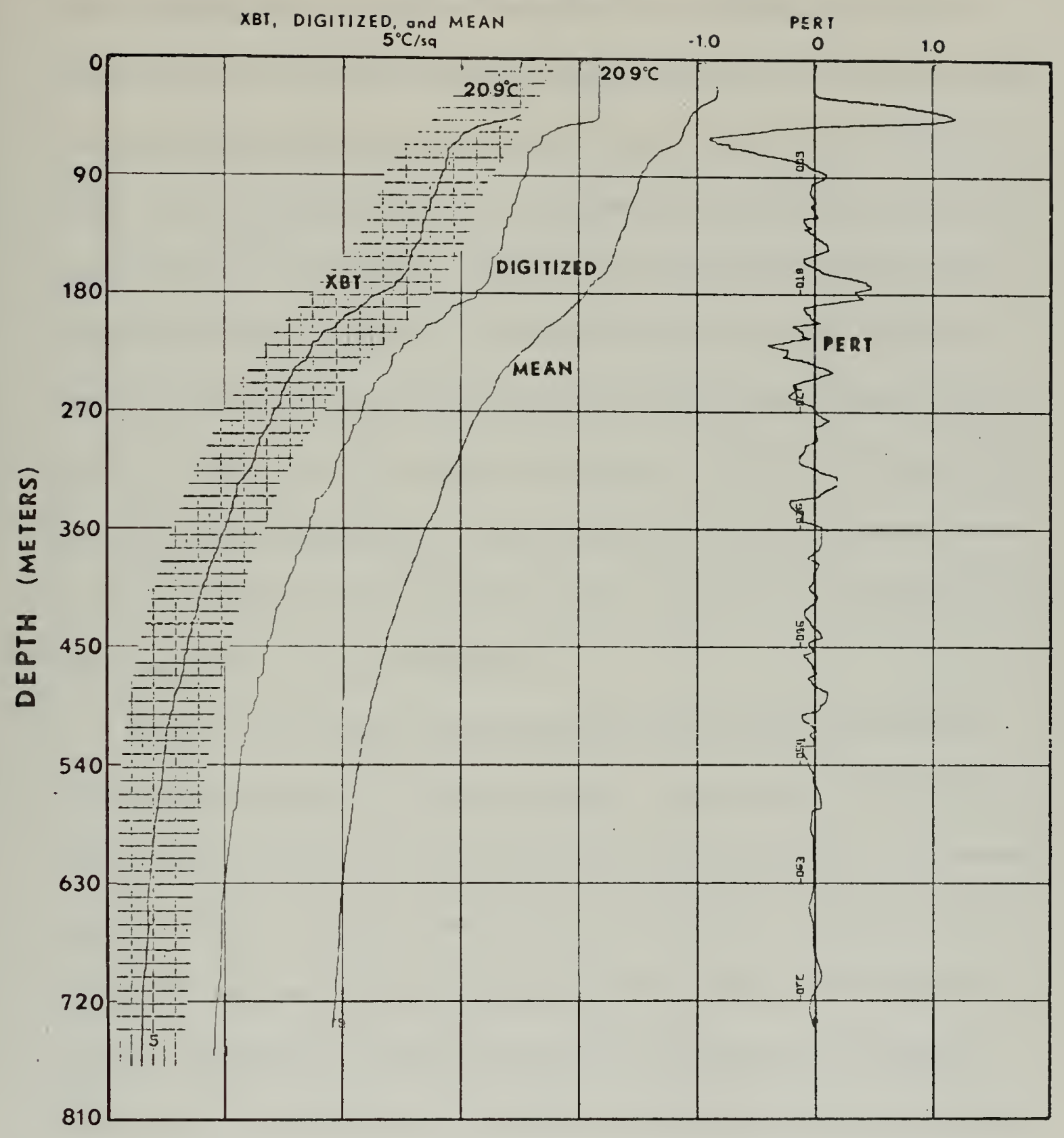

Figure 17. Composite diagram of the original XBT trace, the reconstructed Digitized trace, the Mean curve found by applying Roden's filter, and the Temperature Perturbation curve. The original trace is scaled 92.3 meters/in while the remaining curves are scaled 90.0 meters/in. 

slope changes can be counted and divided into the length of the trace producing a mean temperature perturbation vertical thickness scale which is representative of the microstructure mean vertical scale. The same value can be obtained by dividing by the number of inflection points that was selected for the digitized record. To be sure that only the microstructure is being considered, the inflection points or slope changes above the seasonal thermocline must not be used nor the depth interval from the surface to the seasonal thermocline. This must be determined for each trace independently. The amplitude of the temperature perturbations is defined as the magnitude of the temperature difference between the mean curve and the XBT trace.

Figure $₫ 8$ demonstrates how the temperature perturbations are affected by changing the filtering interval. Examination of the three traces below 100 meters shows that if the filtering interval is increased, then the shape of the perturbation curve is changed. In general, it was found that the mean temperature perturbation vertical thickness scale was increased when the filtering interval $(\Delta \ell)$ was increased, and the temperature amplitude was increased, but not at all depths. It should also be noted that more of the trace is influenced by the mixed layer depth as the interval is increased, so that for the 3 meter curve 150 meters are not useable in computing the mean temperature perturbation vertical thickness scale. 



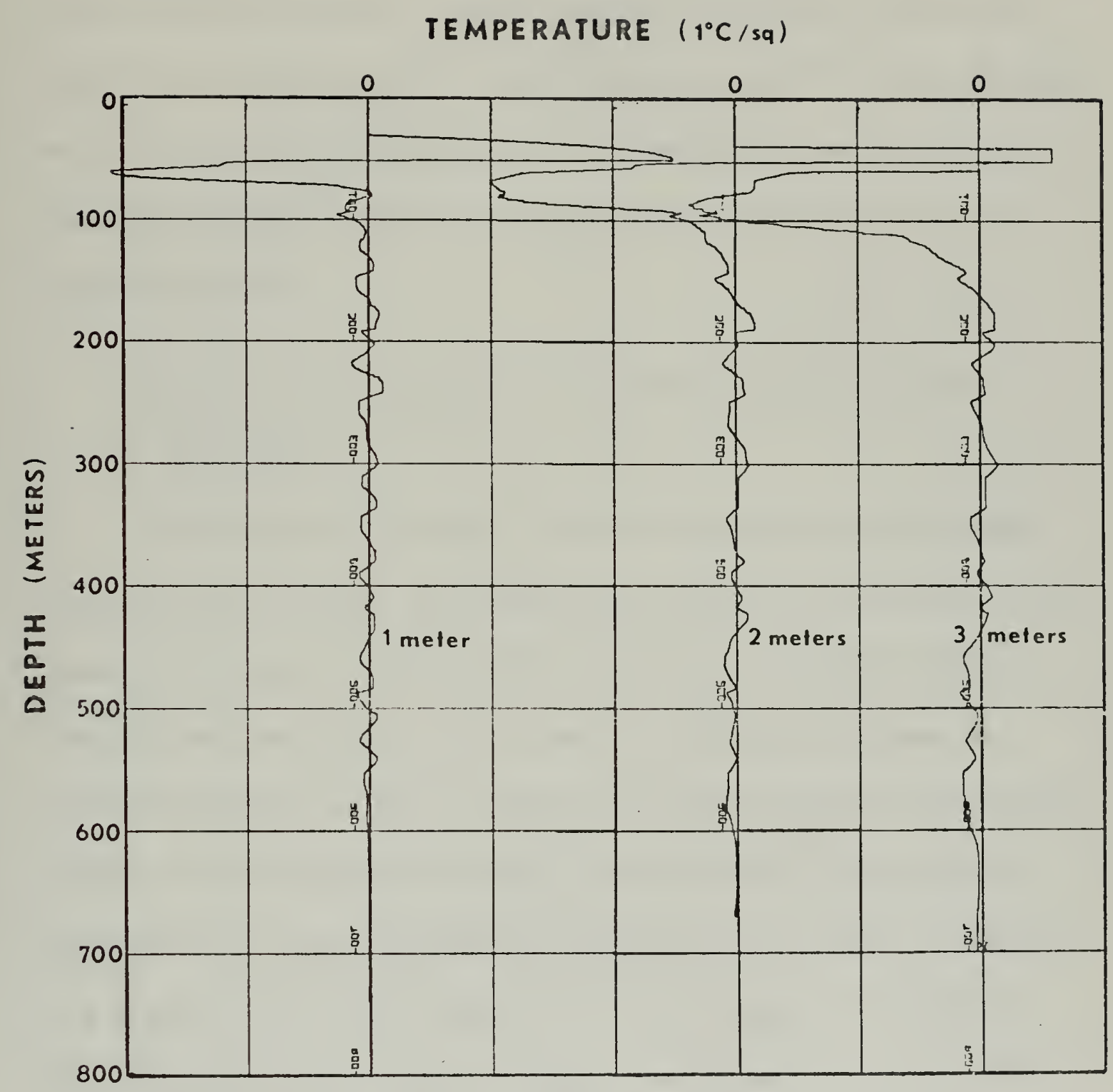

Figure 18. Temperature perturbation curves for a filtering interval of 1,2 , and 3 meters. 

Previous observations of the microstructure indicate scales below a few meters are important. Since increasing the filtering interval produced an increase in the mean temperature perturbation vertical thickness scale, the perturbation amplitude and the variance, it was decided that the 1 meter filtering interval was more effective for processing the digitized XBT data.

\section{G. OCEANOGRAPHIC REGIONS OF THE NORTH PACIFIC OCEAN}

\section{Description}

As previously mentioned, Tully [1964] divided the north Pacific Ocean into seven regions to be used for examining and forecasting oceanographic characteristics and behavior on a similar scale as the Weather Services have been doing for many years. In defining the regions, he considered volume transport, heating and cooling, precipitation and evaporation, mixing (wind and convection), internal waves, and the effects these processes have on changing the properties of sea water. Figure 19 is a diagram of the Pacific Ocean and the seven regions. The Tropic, Subtropic, Arctic, and Subarctic regions are the water mass formation areas where due to slow and/or zonal transport, the water mass has time to establish the regional properties. The normal time required to achieve these properties is one heating and cooling season.

The Tropic region is bounded by Latitudes $10^{\circ} \mathrm{S}$ and $10^{\circ} \mathrm{N}$. . Wind is the dominant mixing factor. The temperature is warm with a small seasonal variation. The flow is generally zonal with a net westward transport. 



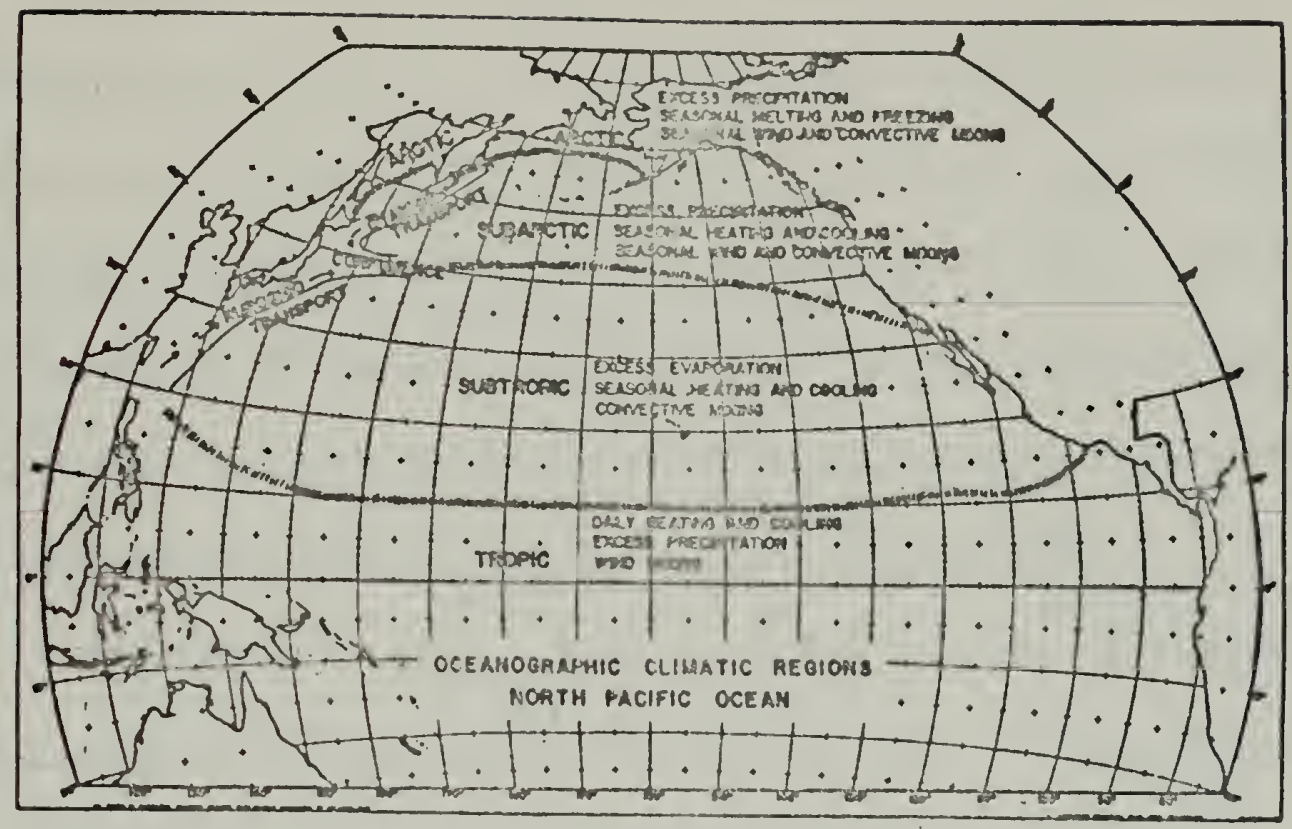

Figure 19. Regions of the North Pacific Ocean [Tully, 1964].

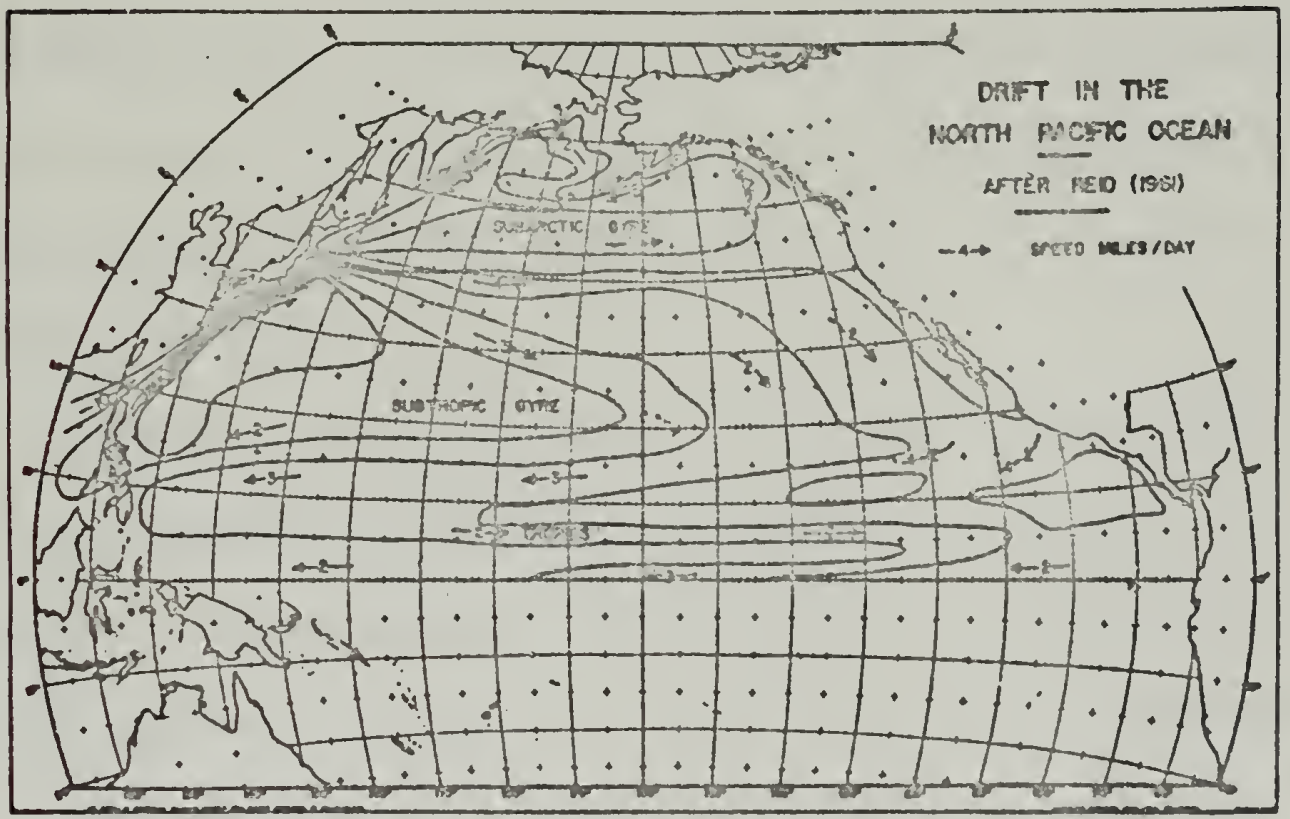

Figure 20. Drift in the North Pacific Ocean [Tully, 1964]. 

The Subtropic is the largest of the regions. The flow is zonal and relatively slow, as is shown in Figure 20. The evaporation exceeds precipitation, causing an evaporation-driven convection mixing. The density structure is primarily a function of the temperature structure. From spring to autumn more heat is added during the day than is lost during cooling at night, so that in September the warmest surface water occurs. The region extends north to between Latitudes $40^{\circ} \mathrm{N}$ and $45^{\circ} \mathrm{N}$.

The Subarctic region is characterized by excess precipitation and as a result there is a distinctive salinity structure. Fresh water accumulates in the upper 100 meters with a halocline in the next 100 meters where salinity increases about 1 percent. The density in this region is primarily a function of salinity. Mixing is due to the wind alone.

The Pacific Arctic region also has an excess of precipitation and a salinity halocline. There is an ice cover in the winter and until midsummer the heat added is used to melt the ice, followed by a rapid and shallow warming due to the long northern days. Convective mixing is dominant in this region.

The Transport regions occur at the western boundaries of the ocean. An intensified meridianal flow transports water between the regions of zonal flow (Figure 20). The water does not remain in these transport regions long enough to fully acquire the regional characteristics. The Kuroshio Current is the western boundary for the Subtropic region. It transports a thick layer of warm saline water formed 

in the Tropics near the Philippine Islands past the coast of Japan losing some of its heat along the way, but never becoming fully adjusted to the climate of the region. Its boundaries are easily identifiea due to large horizontal temperature gradients.

The Oyashio Current is the western boundary for the Subarctic region. It has a smaller rate and volume transport than the Kuroshio Current. The flow is coast hugging and becomes strongest during the summer when it is swelled because of the ice melting in the Arctic.

The Confluence Region is the area where the Kuroshio and Oyashio Currents meet. The resultant flow is eastward. The boundaries between these two flows can have temperature gradients as high as $5^{\circ} \mathrm{C}$ in a mile. Eddies from one stream can be dissolved in the other stream. As the Confluence moves east it looses its identity and is dissipated in the slow eastward trans-ocean flow (Figure 20). More than two years are required for the water to cross from Asia to Amexica [Uda, 1963] so the surface processes can fully re-establish the regional characteristics.

\section{Microstructure Distribution}

The data available to this author necessitated that the regions investigated be restricted to the Subtropic, Subarctic, Kuroshio Current and Confluence regions. The first region and the one most thoroughly examined was the Subtropic for the purpose of comparing the results with 

those of Roden [1971] who had conducted a detailed study of the vertical temperature and salinity structure in the central North Pacific in April 1968 using STD data.

Six locations were selected in the Subtropic region to see if the mean temperature perturbation vertical thickness scale was related within the region. The mean temperature perturbation vertical thickness scale was found for each of the 68 available traces using a 1 meter filtering interval. The average was then found for the traces from each of the six locations. This average mean temperature perturbation vertical thickness scale will be referred to as the average thickness scale. Also for each trace, the maximum amplitude of the temperature perturbation was found and then averaged for each location. Table II presents the results for the Subtropic region. The minimum, maximum, and average values for each location are listed along with the number of traces analyzed for each location. Roden's values are given for similar locations for comparison. The average thickness scale varies between 8.7 and 15.0 meters which compares favorably with Roden's results which show a variation from 13 to 16 meters for this region. One reason that a wide range of variations was found is that in analyzing the data, Roden used zero crossings to compute the thickness scale while in this analysis, inflection points from the digitized record were used because, as was pointed out in Section IV F, they represent layers resolved by the digitizing process. In addition, the 68 traces that were averaged represent all of the months of 

the year and not just April. Another factor is that the maximum depth of the XBT traces was 750 meters while the STD record extended to 1500 meters.

The average maximum amplitude of the temperature perturbations varies between 0.30 to $0.49^{\circ} \mathrm{C}$ which is from the same minimum to three times the maximum value Roden found. This difference is probably largely due to the STD not recording the total magnitude of a temperature change since its response time is about one-third as fast as the XBT. The STD is also temperature compensated in the salinity sensor and large temperature changes in the water column cause a salinity spike that will show by comparison with the temperature record the whole change was not recorded. Another important factor offecting the STD is that it is not free from the vertical motion of the platform. This vertical motion as well as the physical size of the instrument could distort the temperature structure before it is sampled.

Table III gives the results for the Subarctic region. Only 12 traces were analyzed. The presentation is the same as for the Subtropic region with the exception of the omission of the Roden values since he did not have any results from this region. The average thickness scale varied between 17.4 and 82 meters. The location for the maximum thickness scales were in the Gulf of Alaska in the Alaskian gyre. The three traces analyzed were for March, April, and July with a mean thickness scale of $84 \mathrm{~m}, 101 \mathrm{~m}$, and $51 \mathrm{~m}$ respectively. The general behavior (more 

microstructure activity present during the long summer days and less during March, the coldest month) of the conditions is as would be expected since the area is unstable and with the cold winter, convection mixing would tend to homogenize the water column and with the summer heating the microstructure would reform. The average maximum amplitude of the temperature perturbations varied between 0.16 and $0.42^{\circ} \mathrm{C}$.

The Kuroshio Current and Region of Confluence results are shown in Table IV. The only comment on the data is that at $40 \circ \mathrm{N} 149 \mathrm{~F}^{\circ}$ the average thickness scale is smaller than for the rest of the region. This small value indicates a large concentration of microstructure activity and should be expected because this is the area where the Kuroshio and Oyashio currents meet, creating a region of intense vertical shear that is effective in producing microstructure.

Table. $V$ gives results from two other areas. One location was in the Luzon Strait between Luzon and Taiwan. This also demonstrates that there is a large amount of microstructure present and again is in a location that current shear could be expected due to the restriction of flow.

Also in Table $V$ are the results of a 28 trace time series taken on two days in the California Current 200 nautical miles west of Monterey, California. Even though the extremes of the mean thickness scale are widely separated, 19 of the traces or 68 percent were within \pm 2 meters of the average value (13.0 meters). Table VI gives the local time, mean 

thickness scale, and maximum amplitude of the temperature perturbations for each of the 28 traces. The hours are not consecutive because some of the traces were rejected by FNWC, Monterey when received for processing. Without weather information, it is not possible to correlate the microstructure mean thickness scales and the local time.

The average thickness scale data from Tables II, III, IV, and V has been plotted in Figure 21 with the latitude of the location, and shows quite clearly that in the north Pacific Ocean for an increase in latitude the average mean temperature perturbation vertical thickness scale increases. A similar plot, Figure 22, shows that the average maximum amplitude of the temperature perturbations decreases with an increase in latitude. Due to the scatter of data the relationship is not as clear as for the thickness scale. Figure 23 is a plot of the maximum amplitude of the temperature perturbations versus the average mean temperature perturbation vertical thickness scale. Although the scatter of data is wide, the relationship of the plot can be determined. For an increase in the thickness scale of the microstructure the maximum amplitude of the temperature perturbation will decrease. Sverdrup, Johnson, and Fleming [1942] have shown an increase in water instability when proceding north from Hawaii to Alaska. With an increase in instability the thick layers of turbulent convective mixing and of small temperature gradient would become thicker, eliminating the thin layers of high gradient. This is a possible explanation for the results shown in Figure 21. 

Climatological data was not obtained so that no conclusions could be drawn conceming the local time and the microstructure activity. 



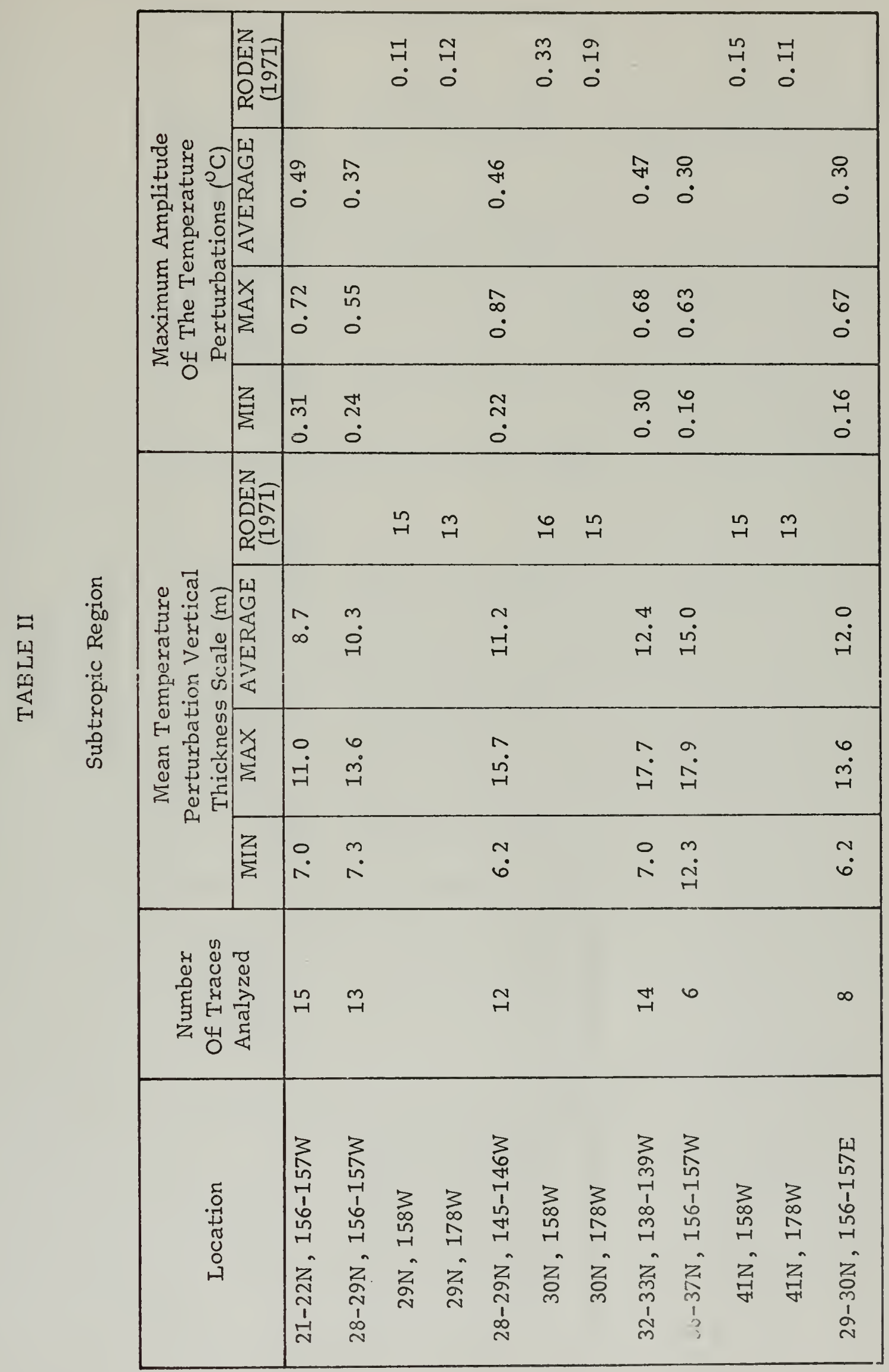





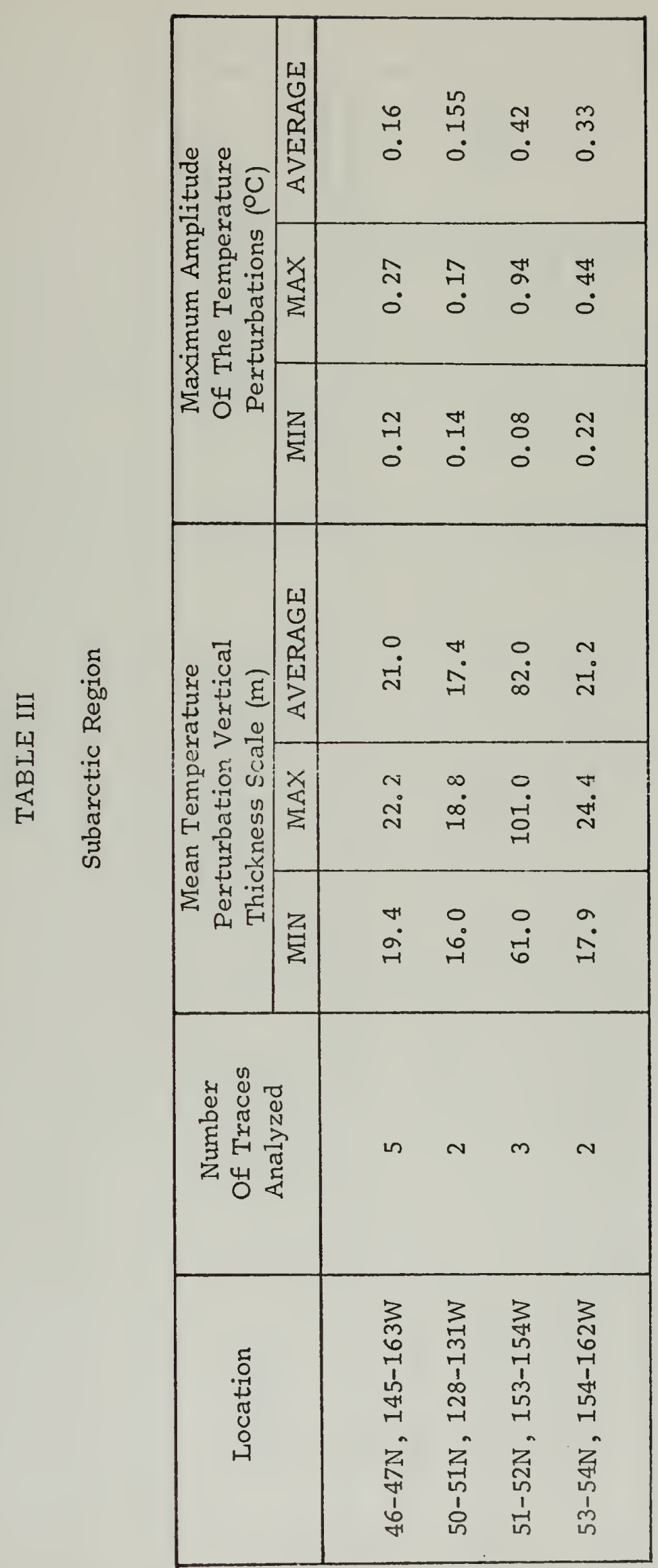





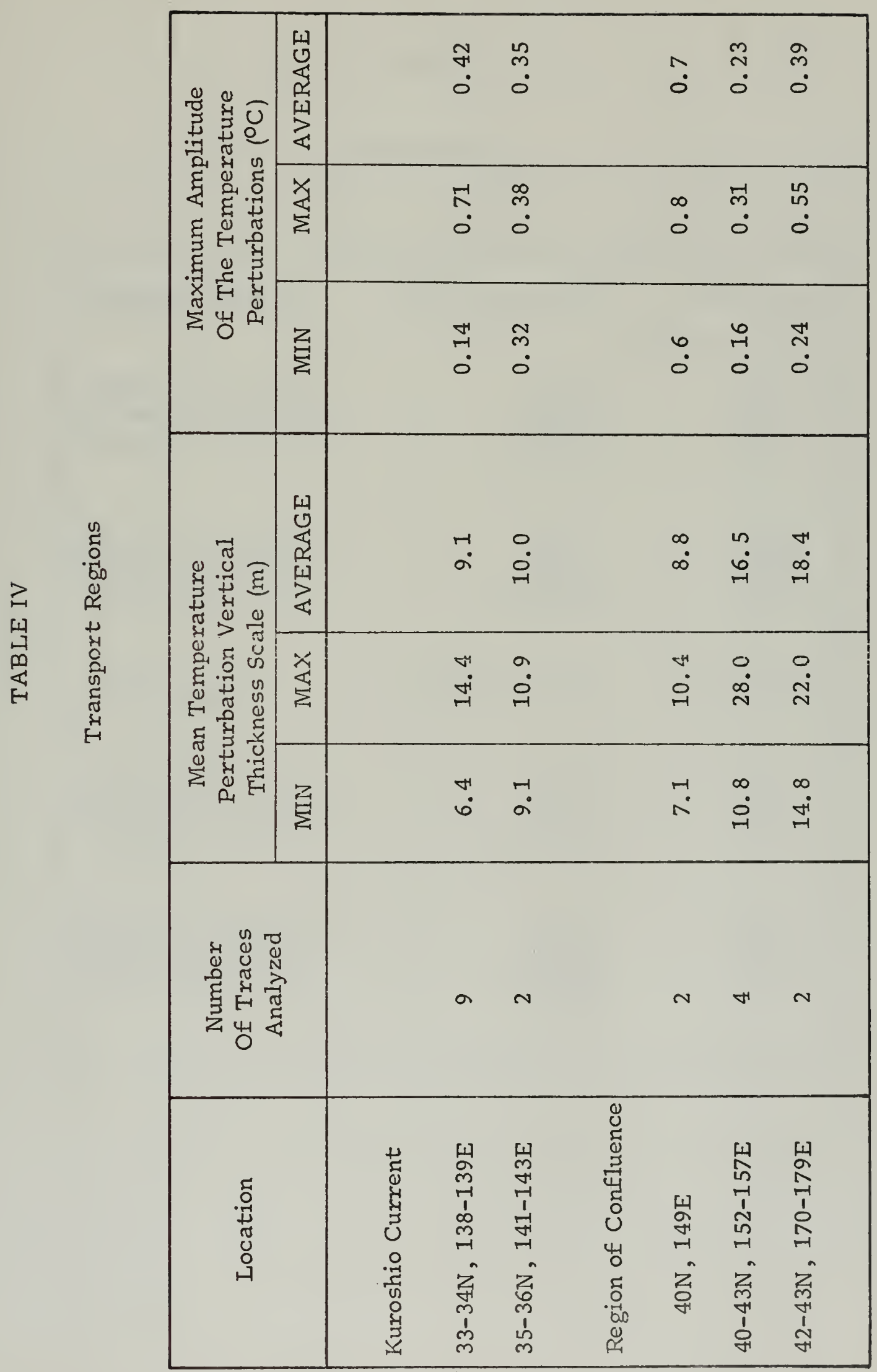



TABLE V

Other Areas

$\begin{array}{ccc}\text { California Current } & 36-37 \mathrm{~N}, 125-126 \mathrm{~W} & 28 \text { traces } \\ & & \\ \text { Mean Temperature } & \text { MIN } & 7.9 \\ \text { Perturbation Vertical } & \text { MAX } & 19.3 \\ \text { Thickness Scale (m) } & \text { AVERAGE } & 13.0 \\ & & \\ \text { Maximum Amplitude } & \text { MIN } & 0.16 \\ \text { Of The Temperature } & \text { MAX } & 0.33 \\ \text { Perturbations }\left({ }^{\circ} \mathrm{C}\right) & \text { AVERAGE } & 0.24 \\ & & \\ \text { Luzon Strait } & & 2 \text { traces } \\ & & \\ \text { Mean Temperature } & \text { MIN } & 6.5 \\ \text { Perturbation Vertical } & \text { MAX } & 7.8 \\ \text { Thickness Scale (m) } & \text { AVERAGE } & 7.15 \\ & & \\ \text { Maximum Amplitude } & \text { MIN } & 0.43 \\ \text { Of The Temperature } & \text { MAX } & 1.00 \\ \text { Pexturbations }\left({ }^{\circ} \mathrm{C}\right) & \text { AVERAGE } & 0.71 \\ & & \end{array}$



California Current Time Series

\begin{tabular}{|c|c|c|c|}
\hline Date & $\begin{array}{l}\text { Local } \\
\text { Time }\end{array}$ & $\begin{array}{l}\text { Mean Temperature } \\
\text { Perturbation Vertical } \\
\text { Thickness Scale }(\mathrm{m})\end{array}$ & $\begin{array}{l}\text { Maximum Amplitude } \\
\text { Of The Temperature } \\
\text { Perturbations }\left({ }^{\circ} \mathrm{C}\right)\end{array}$ \\
\hline \multirow[t]{10}{*}{11 Nov 68} & 0200 & 15.0 & 0.24 \\
\hline & 0400 & 15.4 & 0.22 \\
\hline & 0500 & 12.8 & 0.32 \\
\hline & 0600 & 13.3 & 0.16 \\
\hline & 0700 & 16.6 & 0.20 \\
\hline & 0900 & 13.1 & 0.20 \\
\hline & 1000 & 15.9 & 0.18 \\
\hline & 1100 & 15.0 & 0.33 \\
\hline & 1200 & 10.7 & 0.29 \\
\hline & 1300 & 11.0 & 0.25 \\
\hline 13 Nov 68 & 2300 & 7.9 & 0.20 \\
\hline \multirow[t]{17}{*}{14 Nov 68} & 0100 & 12.5 & 0.22 \\
\hline & 0200 & 8.7 & 0.16 \\
\hline & 0400 & 19.3 & 0.10 \\
\hline & 0500 & 13.5 & 0.16 \\
\hline & 0600 & 15.7 & 0.18 \\
\hline & 0800 & 10.7 & 0.22 \\
\hline & 0900 & 13.1 & 0.20 \\
\hline & 1000 & 9.9 & 0.24 \\
\hline & 1100 & 13.5 & 0.16 \\
\hline & 1200 & 12.9 & 0.14 \\
\hline & 1300 & 11.0 & 0.20 \\
\hline & 1400 & 10.5 & 0.27 \\
\hline & 1500 & 13.3 & 0.20 \\
\hline & 1700 & 13.1 & 0.22 \\
\hline & 1800 & 14.3 & 0.22 \\
\hline & 1900 & 12.8 & 0.26 \\
\hline & 2100 & 12.4 & 0.24 \\
\hline
\end{tabular}





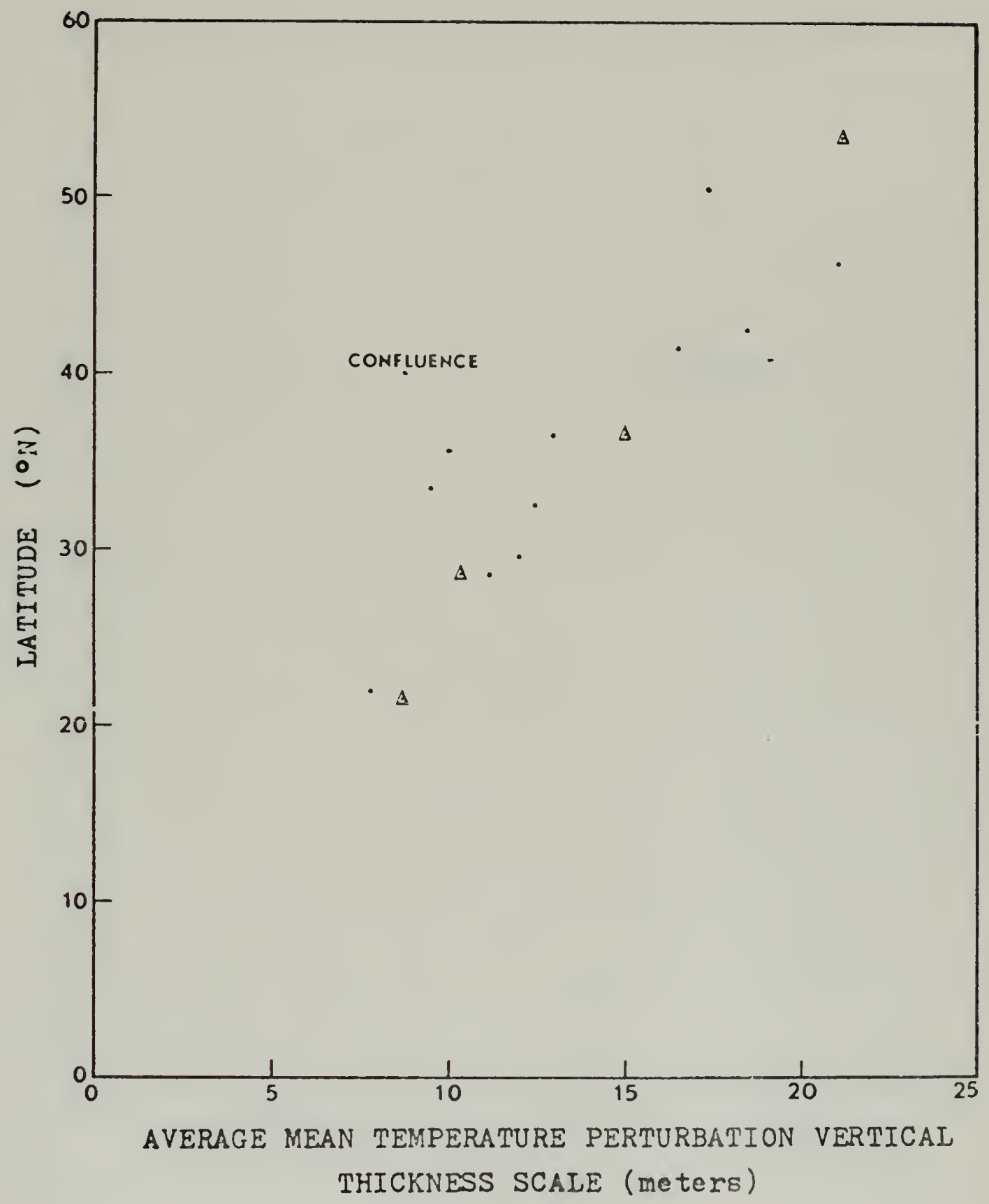

Figure 21. Plot of the Average Mean Temperature Perturbation Vertical Thickness Scale versus Latitude showing an increase in the vertical thickness scale for an increase in latitude between $20^{\circ} \mathrm{N}$ and $55^{\circ} \mathrm{N}$. ( $\Delta$, points along Longitude $156-157^{\circ} \mathrm{W}$; $\cdot$, all other points). 



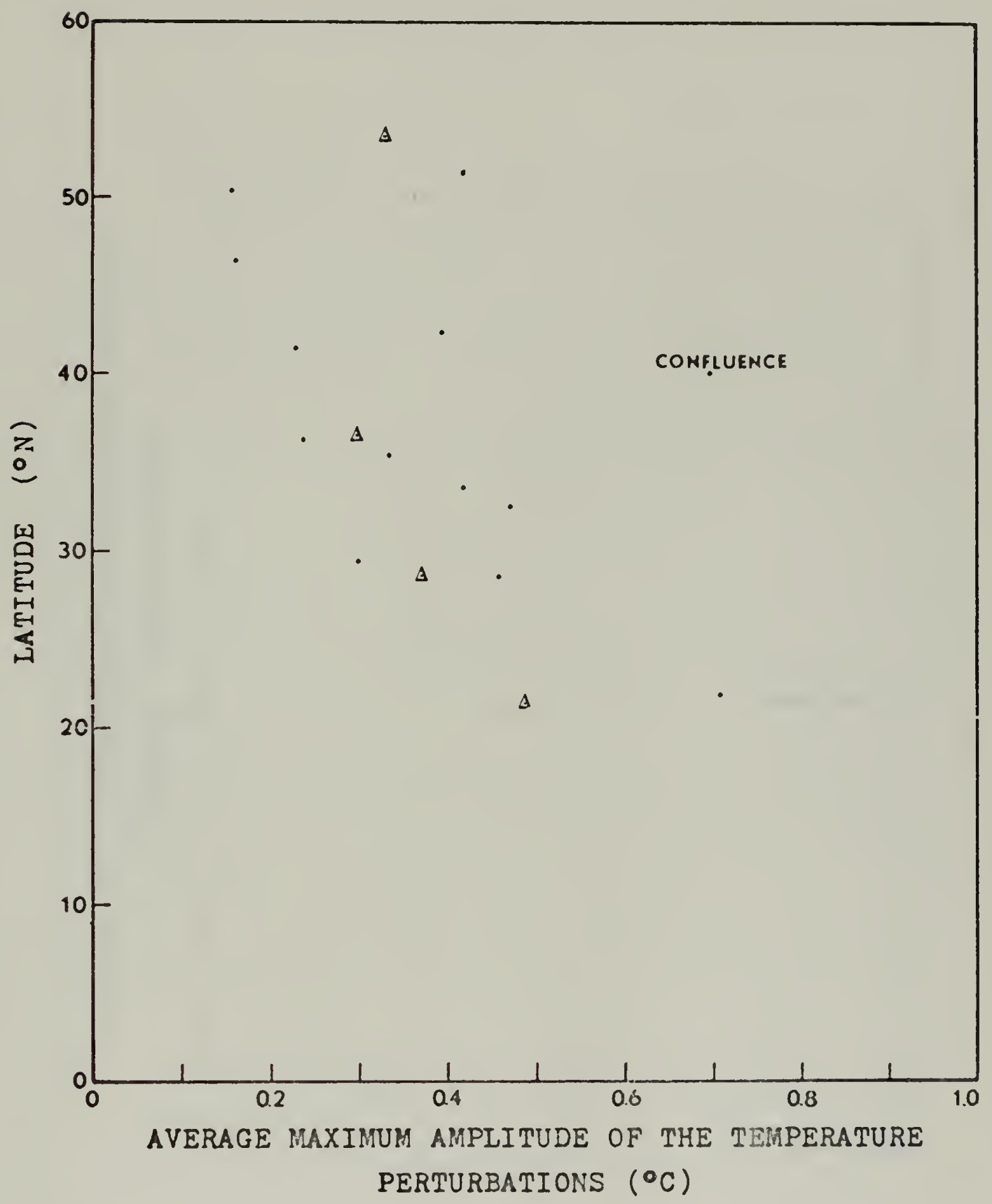

Figure 22. Plot of the Average Maximum Amplitude of the Temperature Perturbations versus Latitude showing the Amplitude decreases with an increase in latitude between $20^{\circ} \mathrm{N}$ and $55^{\circ} \mathrm{N}$. ( $\Delta$, points along Longitude $156-15 \% \mathrm{~W} ; \cdot$, all other points). 



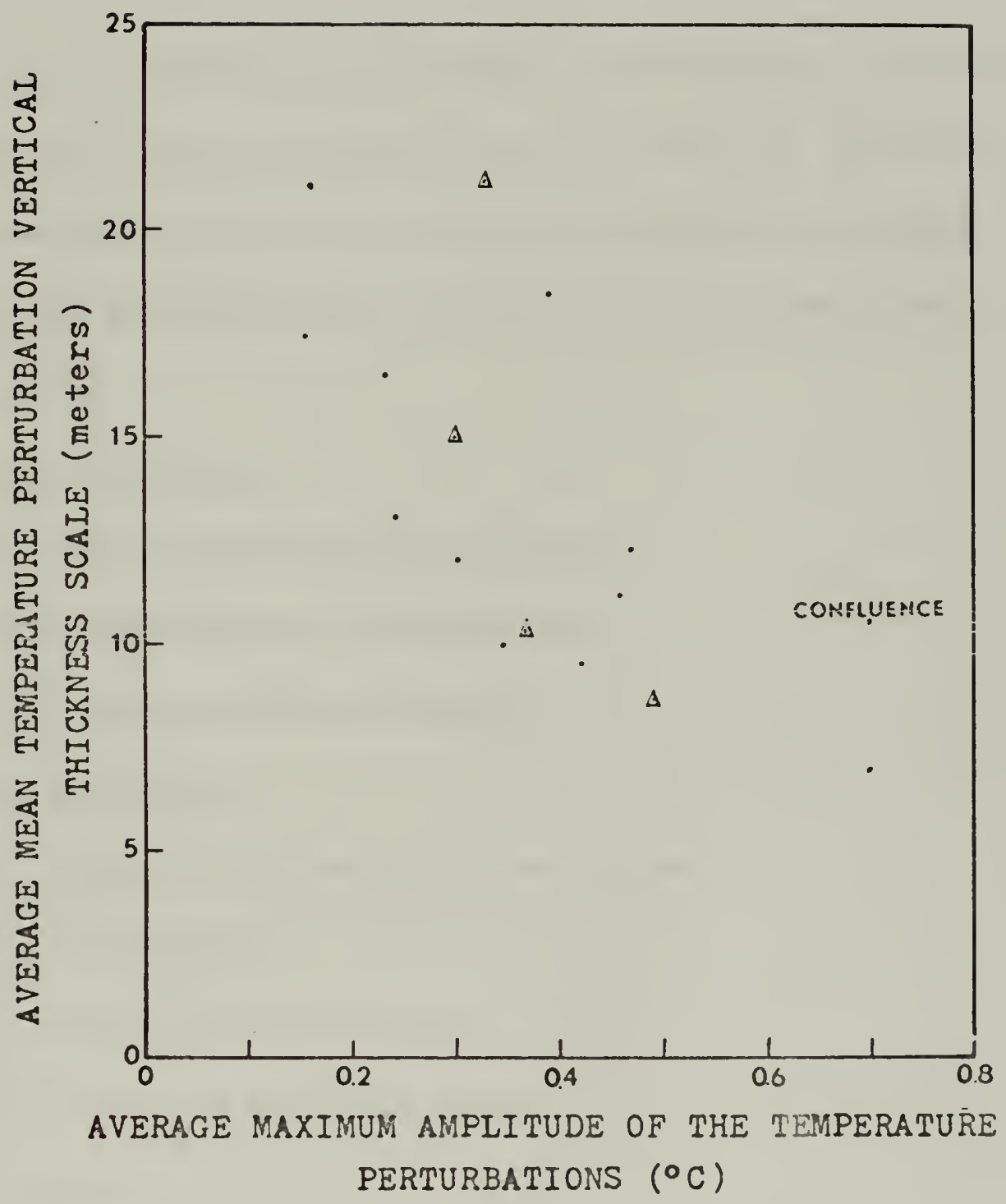

Figure 23. Plot showing the Average Maximum Amplitude of the Temperature Perturbations decreases as the Average Mean Temperature Perturbation Vertical Thickness Scale increases between $20^{\circ} \mathrm{N}$ and $55^{\circ} \mathrm{N}$. ( $A$, points along Longitude $156-157^{\circ} \mathrm{W} ; \cdot$, all other points). 



\section{OPERATIONAL ANALYSIS}

\section{A. OPERATIONAL REQUIREMENT}

Underwater acoustic energy transmissions are extremely vital to the Navy, especially in the anti-submarine warfare (ASW) field. Some of the military uses and some of the non-military uses of underwater sound as reported by the National Academy of Sciences [1970] are listed below by frequency bands.

1. Less than $100 \mathrm{~Hz}$

a. Passive and active long range sonar

b. SOFAR navigation and positiouing

c. Deep exploration and mapping

2. 100 to $5000 \mathrm{~Hz}$
a. Shallow seismic exploration and mapping
b. Warning be11s
c. Scuba diver communications
d. Passive and active ship sonars
e. Mines

3. 5 to $15 \mathrm{kHz}$
a. Underwater telephone transmissions
b. Depth sounding equipment
c. Fish finding
d. High frequency ship sonar 

4. 15 to $100 \mathrm{kHz}$
a. Short range underwater telephone transmissions
b. Depth soundings
c. Fish finding
d. Ship positioning
e. Object location
f. Low resolution scanning sonars
g. High resolution sound velocity profiling
h. Homing torpedoes

5. Greater than $100 \mathrm{kHz}$
a. Doppler sonar for navigation or docking
b. High resolution scanning sonar

For low frequency acoustic transmissions thermal microstructure should not have a significant effect on the propagation because the acoustic wave length is large relative to the effective radius of a patch of thermal inhomogeneities. However, losses at low frequencies have been related to scattering by the index of refraction inhomogeneities [Urick, 1967]. Above a few hundred cycles the microstructure becomes increasingly important and affects sonar transmissions adversely.

Sagar [1960], by taking temperature profiles while collecting acoustic (14. $5 \mathrm{kHz}$ ) transmission data, was able to associate acoustic intensity fluctuations with the presence of microstructure while in the absence of 

microstructure the variation was small and independent of the Mintzer dependence on range. This illustrates the necessity for the Navy to be concerned with thermal microstructure.

\section{B. OPERATIONAL METHODS.}

XBT data is being used by the Navy to obtain the gross temperature profile for use in the solution to ray path equations and for diagrams to predict sonar performance. This can be accomplished on board a ship or can be accomplished by Eleet Numerical Weather Central, Monterey in a more sophisticated manner, and the products supplied as a service to fleet units.

On board ships the parameters most used are the sea surface temperature and layer depth. Tables can then be utilized to find the predictea range for active and/or passive sonar contacts. Little information concerning the temperature structure is presently being used in weapon selection or in determining the expected performance of the weapon once in the environment.

FNWC, Monterey supplies information on request to support ASW operations. The products include sonar range predictions and propagation loss predictions. The major factors which are used for the prediction computations are water mass type, bottom type, and depth of the water. Temperature information is furnished from the historical data bank with no real time input from the area in question. Historical data is used to 

prevent the computer solution from being biased in case the ship XBT was from a small patch of water rather than being a representative sample of the total area. Again, no microstructure information is being used.

\section{POSSIBLE UTILIZATION OF MICROSTRUCTURE INFORIATION ON AN XBT TRACE.}

A ship has the capability of sampling the temperature in its area when desired. However, the XBT trace is not being fully utilized. By closer examination of the trace, the sonar operator could derive an indication of the quantity of microstructure present in his operating area. With this additional knowledge the operator would know whether to expect a variation in sonar reception or to expect steady reception. He would also know of the possibility of picking up contacts beyond the predicted range that might be held only intermittently if microstructure is present. He could request reduced range to maintain contact if the contact is gained at the predicted range and microstructure is present. Use could also be made of microstructure information in selecting the range for launching a homing torpedo. The variation in acoustic intensity will be larger for the frequency range of the homing torpedo than it will be for sonar frequencies, so it would enhance the oppoxtunity for success of the torpedo if the presence or absence of thermal microstructure is considered.

FNWC, Monterey could aid the ASW problem by predicting the relative magnitude of microstructure to be expected for an area by examining the microstructure on digitized traces now in the data bank for the area. This could be made a part of the sonar range prediction now avcilable. 



\section{CONCLUSION}

\section{A. SUMMARY}

The motive behind the investigation of the thermal microstructure distribution in the north Pacific Ocean pursued in this thesis has been to determine what information can be obtained from XBT records and then used to give some prediction of the influence on sonar transmissions and receptions. Various techniques were explored to determine a measure of the thermal microstructure on an XBT trace ending with the selection of a modified binomial filter, suggested by Roden [1971], being preferred. Data was extracted from Fleet Numerical Weather Central, Monterey's XBT data bank, for four of Tully's [1964] climatic regions of the north Pacific Ocean.

\section{B. CONCLUSIONS}

Based on the examination of the data from the Subtropic, Subarctic, Kuroshio Current, and Confluence regions, the following conclusions axe offered:

1. The modified binomial filtex is a technique that will produce a mean temperature pertuxbation vertical thickness scale for the thermal microstructure found on an XBT trace.

2. The digitizing process used by Fleet Numerical Weather Central, Monterey, divides the trace into layers of linear temperaiture gradient 

so that the use of the digitized inflection points will give the same result as that obtained using inflection points from the temperature perturbation curve.

3. The binomial filter will also produce a measure of the amplitude of the temperature perturbations.

4. The average mean temperature perturbation vertical thickness scale increases as latitude increases, while the average maximum amplitude of the temperature perturbations decreases as latitude increases in the north Pacific Ocean between $20 \mathrm{~N}$ and $55 \mathrm{~N}$.

5. The average maximum amplitude of the temperature perturbations decreases if the average mean thickness scale is increased in the north Pacific Ocean.

6. The average mean temperature perturbation vertical thickness scale was found to be more dependent on the latitude than Tully's Climatic Regions.

7. No association could be determined between the time of day or season and the mean thickness scale.

C. RECOMMENDATIONS

The XBT digitized data could be used for a variety of studies. Recommendations for further study enumerated below are specifically related to the development of the data processed in this thesis.

1. One location should be selected for an extensive study to determine if the XBT data will reveal a correlation with time of day and season. 

Two likely areas in the Pacific Ocean for such a study are the San Diego and Hawaiian operating areas used by the Navy. XBT data should be very plentiful from these areas.

2. A study should be conducted to determine the sonar transmission and reception variation that can be expected from the influence of thermal microstructure that appears on XBT traces taken during the study.

3. Fleet Numerical Weather Central, Monterey, Cailifornia, should investigate the feasibility of including a microstructure quantitative prediction as a part of the Sonar Performance forecast for the active sonars.

4. Fleet Numerical Weather Central, Monterey, California, should add to the identification portion of each digitized XBT trace the climatol- gical data, such as wind speed, sea surface temperature, and cloud cover, for ease in evaluation of the trace. 



\section{LIST OF REFERENCES}

1. Bauer, R. A., 1969. Fleet Numerical Weather Central's XBT Digitizing System, Computer Applications Report.

2. Bergmann, P. G., 1946. "Propagation of Radiation in a Medium with Random Inhomogeneities," Physical Review, v. 70, p. 486-492.

3. Choate, T. V., 1970. (Marketing Manager, Sippican Corporation), personal communication.

4. Cohen, J. S., and Weinberg, H., 1969. CONGRATS Ray Plot Program, USL Technical Memorandum 2070-110-69.

5. Cooper, J. W. , and Stomme1, H. , 1968. "Regularly Spaced Steps in the Main Thermocline near Bermuda," Journal of Geophysical Research, v. 73, p. 5849-5854.

6. Cooper, L. H. N., 1967. "Stratification in the Deep Ocean," Sci. Prog. ; v. 55, p. 73-90.

7. Dale, D. H., 1971. (Fleet Numerical Weather Central, Monterey California), personal communication.

8. Dale, D. H., and Stevens, P. D., 1970. Computer Processing of Expendable Bathythermograph Traces, Fleet Numerical Weather Central, Monterey, California,Technical Note 61.

9. Denner, W. W., 1971. "The Layered Microstructure and Acoustic Propagation in the Arctic Ocean," U.S. Navy Journal of Underwater Acoustics, v. 21, p. 45-51.

10. Gouxie, M. W., Sanders, M. R., and Littlehale, A. D. , 1966. Laboratory and Engineering Evaluation of Three Models of Expendable Bathythermographs, USL Report 732.

11. Liebermann, L., 1951. "The Effect of Temperature Inhomogeneities in the Ocean on the Propagation of Sound," Journal of the Acoustical Society of America, v. 23, p. 563-570. 
12. Lovett, J. R. , 1968. "Vextical Temperature Gradient Variations Related to Current Shear and Turbulence," Limnology and Oceanography, v. 13, p. 127-142.

13. Magruder, P. M. , 1970. Some Characteristics of Temperature Microstructure in the Ocean, M. S. Thesis, Naval Postgraduate School, Monterey, California.

14. Miles, J. W., and Howard, L. N., 1963. "Note on a Heterogeneous Shear Flow," Journal of Fluid Mechanics, v. 20, p. $331-336$.

15. Mintzer, D., 1953a. "Wave Propagation in a Randomly Inhomogeneous Medium. I," Journal of the Acoustical Society of America, v. 25, p. 922-927.

16. Mintzex, D., 1953b. "Wave Propagation in a Randornly Inhomogeneous Medium. II," Journal of the Acoustical Society of America, v. 25, p. 1107-1111.

17. Mintzex, D., 1954. "Wave Propagation in a Randomly Inhomogeneous Medium. III " Journal of the Acoustical Suciety of America, v. 26, p. $18 \leqslant-190$.

18. National Academy of Sciences, 1970. Present and Future Uses of Underwater Sound, Washington, D. C.

19. Neshyba, S., Neal, V. T., and Dennex, W. W., 1969. "The Significance of Temperature Stratification in the Arctic," paper presented at Symposium on Military Oceanography, 6 th, Seattle, Washington.

20. Orlanski, I., and Bryan, K., 1969. "Formation of the Thermocline Step Structure by Laxge-Amplitude Internal Gravity Waves," Journal of Geophysical Research, v. 74, p. 6975-6983.

21. Osborn, T. R., 1969. Oceanic Fine Structure, Ph. D. Dissertation, University of California at San Diego.

22. Osborn, T. R., 1970. Report of the Symposium on Turbulence in the Ocean, Institute of Oceanography, University of British Columbia, Vancouver, British Columbia, Manuscript Report No. 24. 

23. Pingree, R. D., 1969. "Small-scale Structure of Temperature and Salinity Near Station Cavall," Deep-Sea Research, v. 16, p. 275-295.

24. Roden, G. I, , 1971. "Spectra of North Pacific Temperature and Salinity Perturbations in the Depth Domain," Journal of Physical Oceanography, v. 1, p. 25-33.

25. Sagax, F. H., 1960. "Acoustic Intensity Fluctuations and Temperature Microstructure in the Sea," Journal of the Acoustical Society of America, v. 32, p. 112-121.

26. Schuert, E. A., 1969. Turbulent Diffusion in the Intermediate Waters of the North Pacific Ocean, Naval Radiological Defense Laboratory Report TR-69-16.

27. Sheehy, M. J., 1950. "Transmission of 24-kc Underwater Sound from a Deep Source," Journal of the Acoustical Society of America, v. 22, p. 24-28.

28. Simpson, J. H., 1971. "Density Stratification in the Western Irish Sea," Deep-Sea Research, v. 18, p. 309$31 \%$.

29. Sippican Corporation, 1968. Instructions for Installation, Operation and Maintenance of Sippican Expendable Bathythermograph System, Sippican Report R-46́7B.

30. Skudrzyk, E., 1957. "Scattering in an Inhomogeneous Medium," Journal of the Acoustical Society of America, v. 29 , p. 50-60.

31. Stern, M. E., 1969. "Collective Instability of Salt Eingers," Journal of Fluid Mechanics, v. 35, p. 209-218.

32. Stommel, H., and Federov, K. N., 1967. "Small Scale Structure in Temperature and Salinity Near Timor and Mindanao," Tellus, v. 19, p. 306-325.

33. Sverdrup, H. U., Johnson, M. W. , and Fleming, R. H., 1942. The Oceans, Prentice-Hall.

34. Tait, R. I., and Howe, M. R., 1968. "Some Observations on Thermo-Haline Stratification in the Deep Ocean," Deep-Sea Research, v. 15, p. 275-280. 

35. Tully, J. P., 1964. "Oceanographic Regions and Assessment of Temperature Structure in the Seasonal Zone of the North Pacific Ocean," Journal of Fisheries Research Board of Canada, v. 21, p. 941-970.

36. Turner, J. S., 1967. "Salt Fingers Across a Density Interface," Deep-Sea Research, v. 14, p. 599-611.

37. Turner, J. S., 1968. "The Behavior of a Stable Salinity Gradient Heated from Below," Journal of Fluid Mechanics, v. 33 , p. $183-200$.

38. Turner, J. S., and Stomme1, H., 1964. "A New Case of Convection in the Presence of Combined Vertical Salinity and Temperature Gradients," Proceedings, National Academy of Sciences, v. 52, p. 49-53.

39. Uda, M., 1963. "Oceanography of the Subarctic Pacific Ocean," Journal of Fisheries Research Board of Canada, v. 20, p. 120-179.

40. Urick, R. J., 1967. Principles of Underwater Sound for Engineers, MCGraw Hill, p. 122-ioo.

41. Whitmarsh, D. C., Skudrzyk, E., and Urick, R. J., 1957, "Forward Scattering of Sound in the Sea and Its Correlation with the Temperature Microstructure," Journal of the Acoustical Society of America, v. 29, p. 1124-1143.

42. Woods, J. D. , 1968a. "Wave-induced Shear Instability in the Summer Thermocline," Journal of Fluid Mechanics v. 32 , p. $791-800$.

43. Woods, J. D. , 1968b. "An Investigation of Some Physical Processes Associated with the Vertical Flow of Heat Through the Upper Ocean," Meteorological Magazine, v. 97, p. 65-72. 
1. The Oceanographer of the Navy

The Madison Building

$732 \mathrm{~N}$. Washington Street

Alexandria, Virginia 22314

2. Commanding Officer

Fleet Numerical Weather Central

Monterey, California 93940

3. Defense Documentation Center

Cameron Station

Alexandria, Virginia 22314

4. Library, Code 0212

Naval Postgraduate School

Monterey, California 93940

5. Professor W. W. Denner, Code 58Dw

Department of Oceanography

Naval Postgraduate School

Monterey, California 93940

6. Professor N. E. J. Boston, Code $58 \mathrm{Bb}$

Department of Oceanography

Naval Postgraduate Schoo1

Monterey, California 93940

7. Department of Oceanography

Naval Postgraduate School

Monterey, California 93940

8. LCDR F. M. Hunt, Jr., USN

USS Corry (DD817)

Fleet Post Office

New York, New York 09501

9. Dr. Ned A. Ostenso

Office of Naval Research

Code 480D

Arlington, Virginia 22217 

10. Mr. J. F. T. Saur

U.S. Department of Commerce

National Oceanic and Atmospheric Administration

National Marine Fisheries Service

Fishery-Oceanography Center

P. O. Box 271

La Jolla, California 92037

11. Mr. Dean Dale

Fleet Numerical Weather Central

Monterey, California 93940

12. Mr. Paul Stevens

1

Fleet Numerical Weather Central

Monterey, California 93940

13. Mr。J. R. Clark

1

Fleet Numerical Weather Central

Monterey, California 93940 
Unclassified

Securty Classification

\section{DOCUMENT CONTROL DATA.R \& D}

isecurity classilication of fille, bodv of abstract and indexing annotation niust be encered when the overall repart is rlassilied) ORIGINATING ACTIVITY (Corporale author)

Naval Postgraduate School

Monterey, California 93940

Unclassified

3 REPORT TITLE

An Examination of Microthermal Structure Statistics as Calculated from

Expendable Bathythermograph Records

DESCRIPTIVE NOTES (TYPe of repori and.inclusive dales)

Master's Thesis; (September 1971)

$s$ aUTHORISI (First name, middle initial, last namo)

Frank M. Hunt, Jr.

\begin{tabular}{|c|c|c|}
\hline $\begin{array}{l}\text { B. AEPORTOATE } \\
\text { September } 1971\end{array}$ & $\begin{array}{l}\text { 7.. TOTAL NO. OFPAGES } \\
83\end{array}$ & $\begin{array}{l}\text { 76. NO. OF REFS } \\
43\end{array}$ \\
\hline $\begin{array}{l}\text { 8a. CONTRACT OR GRANT NO } \\
\text { B. PROJECT NO. }\end{array}$ & \multicolumn{2}{|c|}{ 88. ORIGINATOR.S REPORT NUMBERIS) } \\
\hline c. & $\begin{array}{l}\text { Ob. OTHER REPOAT NO(S) } \\
\text { (his report) }\end{array}$ & her numbers that may be ansigned \\
\hline
\end{tabular}

10. OISTRIBUTION STATEMENT

Approved for public release; distribution unlimited

11. SUPPLEMENTARY NOTES

12. SPONSORING MILITARY ACTIVITY

Naval Postgraduate School

Monterey, California 93940

13. ABSTAACT

Expendable Bathythermograph (XBT) temperature depth profile data from 17 locations in the north Pacific Ocean has been analyzed to extract information concerning the thermal microstructure. The microstructure information is then examined to determine if the distribution can be associated with the climatic regions of the north Pacific Ocean proposed by Tully [1964].

A modified binomial filter used by Roden [1971] to analyze STD data was appied to find a mean temperature series. A temperature perturbation series results when the mean is subtracted from the original XBT series. Two resulting parameters, average mean temperature perturbation vertical thickness scale and average maximum amplitude of the temperature perturbations, can be associated with the microstructure.

The average mean temperature perturbation vertical thickness scale was found to increase with latitude from $20^{\circ} \mathrm{N}$ to $55^{\circ} \mathrm{N}$, and as the thickness scale increased, the maximum amplitude of the perturbations was found to decrease. 
Unclassified

Securitv Classification

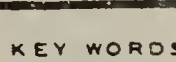

LINK

Microstructure

Microthermal structure

Expendable Bathythermograph

XBT

Digitizing

Temperature

Underwater Sound

Climatic Regions

Temperature Perturbations 







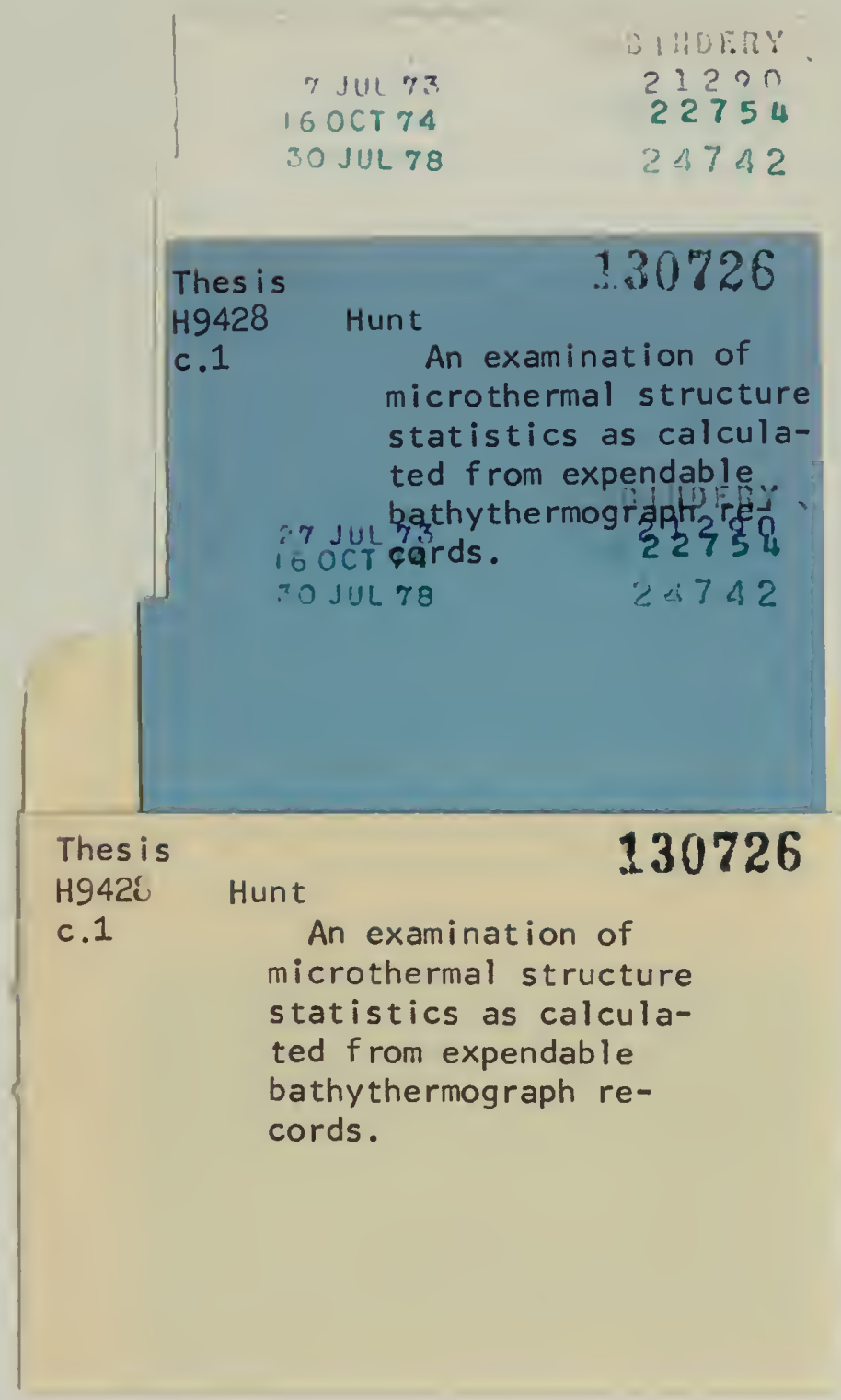




\section{thes $\mathrm{H} 9428$}

An examination of microthermal structure

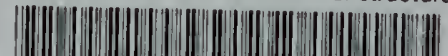

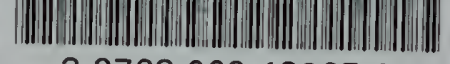

32768002132656

DUDLEY KNOX LIBRARY 